\title{
Learned predictiveness effects following single-cue training in humans
}

\author{
M. E. Le Pelley, M. N. Turnbull, S. J. Reimers, And R. L. Knipe \\ Cardiff University, Cardiff, Wales
}

\begin{abstract}
The results of several recent studies of human associative learning indicate that people will learn more rapidly about cues that have previously been experienced as predictive of events of significance, as compared with cues previously experienced as nonpredictive. Notably, however, these experiments have typically established this prior predictiveness by means of pretraining with multiple, simultaneously presented cues, some of which are more predictive than others. The present experiments instead investigated the influence of prior predictiveness on future learning when this predictiveness was established via pretraining with individual cues, each of which was the best available predictor of the outcome with which it was paired. Results indicate that, following this pretraining, human participants again show better learning about previously predictive cues than about previously nonpredictive cues.
\end{abstract}

One of the primary aims of associative learning theory and the research on which it is based is to establish the factors that influence learning - the factors that determine why, under a certain set of circumstances, an organism might learn more about the consequences of Stimulus $X$ than about those of Stimulus Y. Evidence from the field of animal conditioning suggests that one such factor is the prior predictive history of a stimulus. In particular, previous experience of a cue (e.g., a tone) as being predictive or nonpredictive of an outcome event (e.g., food) will influence the rate of subsequent learning about that cue (e.g., Bennett, Wills, Oakeshott, \& Mackintosh, 2000; Dopson, Esber, \& Pearce, in press; Hall \& Pearce, 1979, 1982; Haselgrove, Esber, Pearce, \& Jones, in press; Lubow \& Moore, 1959; Mackintosh \& Little, 1969; Mackintosh \& Turner, 1971; Wilson, Boumphrey, \& Pearce, 1992; see Le Pelley, 2004, for a review). And consistent with the suggestion that a common associative-learning mechanism might underlie animal conditioning and human contingency learning (Dickinson, 2001), several recent studies have demonstrated an influence of prior predictiveness on the rate of learning about cues in human learning (e.g., Bonardi, Graham, Hall, \& Mitchell, 2005; Griffiths \& Le Pelley, 2009; Kruschke, 1996; Le Pelley \& McLaren, 2003; Le Pelley, Schmidt-Hansen, Harris, Lunter, \& Morris, 2010; Livesey \& McLaren, 2007; Lochmann \& Wills, 2003; Maes, Damen, \& Eling, 2004). This rate of learning about the cue is often referred to as the cue's associability, or, perhaps more controversially, the attention paid to the cue. In this article, we remain uncommitted on exactly what aspect of cue processing is influenced by previous experience of predictiveness - specifically, whether such differences in processing truly reflect differences in atten- tion (see Le Pelley, in press-a). Hence, from this point on, we will use the theoretically neutral label alpha to refer to that property of cue processing that is influenced by the prior predictiveness of the cue.

The problem posed by the literature cited above is that the specific way in which the learned predictiveness of stimuli influences alpha seems very inconsistent. With regard to the animal learning literature, the results of certain studies indicate that stimuli that have previously been experienced as reliable predictors will maintain a higher alpha (and hence, will subsequently be learned about more rapidly) than will stimuli experienced as nonpredictive (Bennett et al., 2000; Dopson et al., in press; George \& Pearce, 1999; Mackintosh, 1969; Mackintosh \& Little, 1969). Such findings fit well with the theoretical stance taken by Mackintosh (1975; hereafter, referred to as the Mackintosh model; see also Kruschke, 2001; Sutherland \& Mackintosh, 1971). Other studies, however, seem to demonstrate exactly the opposite, showing faster learning about stimuli that have previously been unreliable predictors, as compared with those experienced as consistently predictive (e.g., Haselgrove et al., in press; Swan \& Pearce, 1988; Wilson et al., 1992). The latter findings fit well with the theory offered by Pearce and Hall (1980; hereafter referred to as the Pearce-Hall model).

Notably, although both the Mackintosh and Pearce-Hall theories assume that the alpha of a stimulus is influenced by the prior predictiveness of that stimulus, they differ in the way that predictiveness is assessed. The Mackintosh model determines alpha on the basis of a comparison of the relative predictiveness of simultaneously presented cues - that is, whether Stimulus $\mathrm{X}$ is a better or worse predictor of a particular outcome than is Stimulus Y. The

M. E. Le Pelley, lepelleyme@cf.ac.uk 
stimulus selected as the best predictor of the outcome then maintains a high alpha, whereas the alpha of all other stimuli decreases. The Pearce-Hall model, in contrast, is based on absolute predictiveness - that is, how well Stimulus X predicts a particular outcome. Pearce and Hall (1980) argued that it makes sense for an animal to devote more learning resources to stimuli whose consequences are still unknown - those with low absolute predictiveness - as compared with those whose consequences are already well established.

Le Pelley (2004; see also Haselgrove et al., in press; Le Pelley, in press-b; Pearce \& Mackintosh, in press) has demonstrated that this distinction in the way that predictiveness is assessed allows a hybrid model, in which both Mackintosh and Pearce-Hall mechanisms interact to determine the overall alpha of a stimulus, to anticipate positive transfer of alpha under some circumstances and negative transfer under others. According to this approach, whether a given experiment demonstrates positive transfer of alpha (faster learning about stimuli previously established as good predictors) or negative transfer of alpha (faster learning about stimuli previously established as poor predictors) will be determined by whether the procedure of that experiment emphasizes relative predictiveness or absolute predictiveness, respectively. In particular, a Mackintosh-like relative predictiveness mechanism should dominate when predictiveness is established during a pretraining phase that involves multiple simultaneously presented stimuli, some of which are more predictive than others. Under these circumstances, a relative predictiveness mechanism will select which of the several available stimuli is the best predictor of reinforcement and will guide learning resources toward that stimulus at the expense of others; hence, we should expect positive transfer of alpha. In contrast, negative transfer of alpha is anticipated when pretraining involves only a single cue, with that cue always being the best available predictor of reinforcement (although exactly how predictive it is might vary between different groups). Under these conditions, the contribution of the relative predictiveness mechanism will be minimized, since, in all cases, it will pick out the same cue as the one on which to focus learning. Consequently, such single-cue learning preparations should, instead, emphasize the absolute predictiveness mechanism of the Pearce-Hall model: Given that the single cue has been "selected," how much remains to be learned about this cue? If a great deal remains to be learned (i.e., the cue has low absolute predictiveness), a Pearce-Hall mechanism will afford a higher learning rate to that selected cue than if little remains to be learned.

Notably, recently reported studies of alpha effects in human contingency learning have established predictiveness in a pretraining phase involving multiple simultaneously presented cues, some of which were better predictors than others (e.g., Bonardi et al., 2005; Griffiths \& Le Pelley, 2009; Kruschke, 1996; Le Pelley \& McLaren, 2003; Livesey \& McLaren, 2007; Lochmann \& Wills, 2003; Maes et al., 2004). These are exactly the circumstances under which, as was argued above, we might expect the relative predictiveness mechanism of the Mackintosh model to dominate; consistent with this approach, all of these studies have yielded results indicating positive transfer of alpha. This leads naturally to the question of whether pretraining human participants with separately presented individual cues (thereby minimizing any influence of relative predictiveness) would, instead, produce results consistent with the Pearce-Hall model - namely, more rapid learning about previously nonpredictive cues than about previously predictive cues, indicating negative transfer of alpha. This idea formed the focus of the present experiments.

The present experiments provide a human analogy to a study by Wilson et al. (1992, Experiment 1). In Wilson et al.'s study, rats in Group Control received pretraining in which a light was consistently paired with a tone. For rats in Group Experimental, the light-tone relationship was inconsistent: Half of the presentations of the light were followed by the tone, whereas the other half were not. Note that, for both groups, the light was the best (and only) available predictor of the tone and, hence, did not differ in its relative predictiveness. Consequently, on the basis of Le Pelley's (2004) analysis, we would expect the influence of the Pearce-Hall absolute predictiveness mechanism to dominate, promoting more rapid learning about inconsistent predictors than about consistent predictors. In line with this suggestion, in a subsequent training phase in which the light was paired with food, acquisition of the light-food association proceeded more rapidly in Group Experimental than in Group Control. The present experiments followed this basic approach of pretraining certain stimuli as consistent predictors and others as inconsistent predictors of "neutral" outcomes (although this predictiveness manipulation was within subjects, rather than between subjects, in Wilson et al.'s study [1992]: this issue is discussed further in the General Discussion section), using human participants. We then measured the rate at which the participants learned novel discriminations regarding each class of cue.

\section{EXPERIMENT 1}

Table 1 shows the design of Experiment 1. On each Stage 1 trial, the participants were asked to predict which of two background colors (pink or orange) a particular company had used for a batch of business cards. Letters A-Y in Table 1 represent different company names. Each Stage 1 training block comprised each of the 12 trial types shown in Table 1; thus, each company appeared twice in each block. Companies A-D were consistently paired with the same color throughout Stage $1-\mathrm{A}$ and $\mathrm{D}$ with pink, $\mathrm{B}$ and $\mathrm{C}$ with orange. Cues $\mathrm{A}-\mathrm{D}$ are henceforth referred to as $\mathrm{P}$ (for predictive) cues. In contrast, Companies X and $\mathrm{Y}$ were inconsistent predictors; in each block, each company was paired once with pink and once with orange. Cues $\mathrm{X}$ and $\mathrm{Y}$ are therefore referred to as NP (for nonpredictive) cues. ${ }^{1}$ Note that all the cues were trained individually; there was only one company name presented on each trial. Hence, the contribution of relative predictiveness processes, which operate by selecting which of several simultaneously presented cues best predicts the current outcome, should be minimized. 
Iable 1

Design of Experiment 1

\begin{tabular}{lccc}
\hline \multicolumn{2}{c}{ Stage 1 } & Stage 2 & Test \\
\hline $\mathrm{A} \rightarrow$ Pink & $\mathrm{A} \rightarrow$ Pink & $\mathrm{A}+$ & 1. A vs. B \\
& & & 2. C vs. D \\
$\mathrm{B} \rightarrow$ Orange & $\mathrm{B} \rightarrow$ Orange & $\mathrm{B}-$ & 3. X vs. Y \\
& & & 4. A vs. $\mathrm{X}$ \\
$\mathrm{C} \rightarrow$ Orange & $\mathrm{C} \rightarrow$ Orange & $\mathrm{C}+$ & 5. C vs. $\mathrm{X}$ \\
$\mathrm{D} \rightarrow$ Pink & $\mathrm{D} \rightarrow$ Pink & $\mathrm{D}-$ & 6. B vs. $\mathrm{Y}$ \\
& & & 7. D vs. $\mathrm{C}$ \\
$\mathrm{X} \rightarrow$ Pink & $\mathrm{X} \rightarrow$ Orange & $\mathrm{X}+$ & 8. A vs. C \\
& & & 9. B vs. D \\
\hline
\end{tabular}

On each Stage 2 trial, the participants were told that they had invested in a company and had to predict whether they would make a profit or a loss. A "+" symbol in Table 1 indicates that investment in a company was generally profitable, whereas a "-" symbol indicates that investment was generally loss-making.

The question of interest was the rate at which the participants would learn about profitability during Stage 2. The objective statistical relationship with profitability was identical for companies that had been predictive of card color in Stage 1 and those that had been nonpredictive. Thus, Companies A and C (P cues) were paired with the same majority of profits as was Company X (an NP cue), and Companies B and D were paired with the same majority of losses as was Company Y. Hence, any difference in the rate of learning about these cues would have to result from differences in their learned predictiveness with regard to Stage 1 colors. On the basis of the parallel with Wilson et al.'s (1992) animal study, we might expect more rapid learning about NP cues than about P cues. This learning was assessed using the participants' responses during Stage 2 and, also, in a final test phase, in which they were asked to decide in which of two named companies they would rather invest.

It is important to note that profitability during Stage 2 was statistically independent of card color during Stage 1. That is, of the companies that produced pink business cards in Stage 1, half were generally profitable and half were loss making; the same was true for companies producing orange cards. This prevents explanation of any difference in the extent of Stage 2 learning about $P$ cues versus NP cues in terms of an outcome-mediated associative process of acquired distinctiveness or acquired equivalence (Hall, 1991; Hall, Mitchell, Graham, \& Lavis, 2003). The argument runs thus. Suppose that all the trials with Cues C and $\mathrm{D}$ were omitted from the design shown in Table 1. Stage 1 training would ensure that presentation of Cue A tended to activate the representation of pink. When the participant learned to respond profit on presentation of A in Stage 2, the activated representation of pink would also become associated with profit. Likewise, when the participant learned to respond loss on presentation of B, the representation of orange (B's associate from Stage 1) would become associated with loss. Although association of pink with profit and orange with loss would aid the learning of a Stage 2 discrimination between A and B, it would not aid Stage 2 discrimination between the NP Cues X and $\mathrm{Y}$, since each of the latter cues would activate the representations of pink and orange equally during Stage 2 . Hence, if this were the full design of the experiment, this outcome-mediated acquired distinctiveness mechanism would predict better learning of the Stage 2 discrimination between $\mathrm{A}$ and $\mathrm{B}$ than between $\mathrm{X}$ and $\mathrm{Y}$, even if there were no difference in the alpha of these cues.

Inclusion of Cues C and D ruled out this possibility, however. These cues ensured that, in Stage 2, the associatively activated representations of each Stage 1 outcome would occur equally often in the presence of profit and loss. That is, A would activate pink in the presence of profit, but $\mathrm{D}$ would activate pink in the presence of loss; likewise, B would activate orange in the presence of profit, but $\mathrm{C}$ would activate orange in the presence of loss. Consequently, there would be no way for these associatively activated representations to selectively affect formation of the Stage 2 discriminations. The implication is that any observed difference in the extent to which these discriminations are acquired would reflect a difference in the alphas of the cues involved.

\section{Method}

Participants and Stimuli. Thirty-two Cardiff University students participated in exchange for either $£ 5$ or course credit. They were tested in groups of up to 3 using standard PCs. The six company names were Stonedge, Hedgend, Woodrow, Cornfield, Lakeside, and Maylawn. These were randomly and independently assigned to letters $\mathrm{A}-\mathrm{Y}$ in the experimental design for each participant.

Procedure. The instructions informed the participants that each of the companies had produced several batches of business cards and that, on each trial, they would be asked to decide which of two background colors the named company had used for a particular batch. On each trial, the message "Which color did [company name] use for this batch of business cards?" appeared above pictures of two cards, each bearing the name of the company listed at the top of the screen and differing only in their background color-one pink, the other orange. Whether the pink card appeared to the left or the right of the orange card was determined randomly for each participant but remained constant across Stage 1. The participants entered their decision by clicking the appropriate card. Immediate feedback was provided: A blue box highlighted the correct answer, and if the participants made a correct prediction, the word "Correct" appeared; if they made an incorrect prediction, the word "Wrong" appeared, and a beep was heard over headphones. Each of the 12 trial types shown in Table 1 occurred once per block of Stage 1 in random order, with 
the constraint that there could be no immediate repetitions across blocks. Due to a programming error, 19 participants received four blocks of Stage 1 training, and 13 received six blocks.

Following Stage 1, the instructions informed the participants that, on each trial, they would be told that they had invested in a company and must predict whether they would make a profit or a loss. On each trial, the message "You have invested in [company name]. What do you think will happen?" appeared above a horizontal scale marked with 21 gradations. The leftmost end of the scale was labeled "sure to make a loss," the middle was labeled "profit or loss equally likely," and the rightmost end was labeled "sure to make a profit." The participants entered their decision by clicking at the appropriate point on the scale to set the position of a marker (the scale actually had 41 points; the participants could position the marker midway between two gradations) before clicking an OK button to continue. Immediate feedback was provided: If this was a profit trial, the message "You made a profit" appeared in green; if it was a loss trial, the message "You made a loss" appeared in red. Stage 2 comprised 10 blocks, with each of the six trial types shown in Table 1 appearing once per block in random order, with the constraint that there could be no immediate repetitions across blocks. For cues shown with the "+" symbol in Table 1, on eight presentations the cue was paired with profit, and on two presentations with loss. For cues shown with the "-" symbol, on eight presentations the cue was paired with loss, and on two with profit. On the first two presentations of each cue, the outcome was always of the majority type for that cue. For example, on its first two presentations, Cue A was paired with profit, with the latter eight presentations of Cue $\mathrm{A}$ involving five profit and three loss trials in random order.

After Stage 2, the participants were told that, in the following stage, on each trial they were to imagine that they had $£ 1,000$ to invest; two companies would be presented on the screen, and they were to select which they would rather invest in (i.e., which would be more likely to make a profit in the future) by clicking on the name of that company. There were nine different test comparisons, as shown in Table 1; for example, "A vs. B" indicated a choice between Companies A and B. Each of the nine comparisons appeared once during the test phase, in random order.

Data analysis and exclusion. We could hope to observe differences in learning during Stage 2 only if the participants were indeed able to learn about profitability during this stage. In a minority of cases, the participants' data instead indicated that they had not learned anything during Stage 2, with responding seemingly random. A criterion for data exclusion in this and all subsequent experiments was therefore adopted as follows. If the correct answer on a given Stage 2 trial was profit and a participant gave a prediction rating greater than 20 (the midpoint of the scale), that answer was scored as correct; if the rating was 20 or less, the answer was scored as incorrect. Likewise, if the correct answer on a given Stage 2 trial was loss and a participant gave a rating less than 20 , that answer was scored as correct; if the rating was 20 or more, the answer was scored as incorrect. Data from the first block of Stage 2 were not considered in this assessment of learning, on the assumption that these responses must be guesses (but see Experiment 4). Averaged across the remaining blocks of Stage 2, 8 participants gave correct ratings on $50 \%$ or fewer of the trials - that is, their accuracy remained at or numerically below chance. The data from these participants were therefore excluded from further analyses. Significance in all the analyses reported below was assessed against a Type I error rate of $\alpha=.05$.

\section{Results and Discussion}

Figure 1 shows the mean percentages of correct responses per block across the six blocks of Stage 1. As was expected, accuracy increased rapidly for $\mathrm{P}$ cues as the participants learned the correct responses but remained low for NP cues. Figure 1 reveals that accuracy of responses to NP cues actually remained slightly below the chance level of 50\% throughout Stage 1. A one-tailed $t$ test using

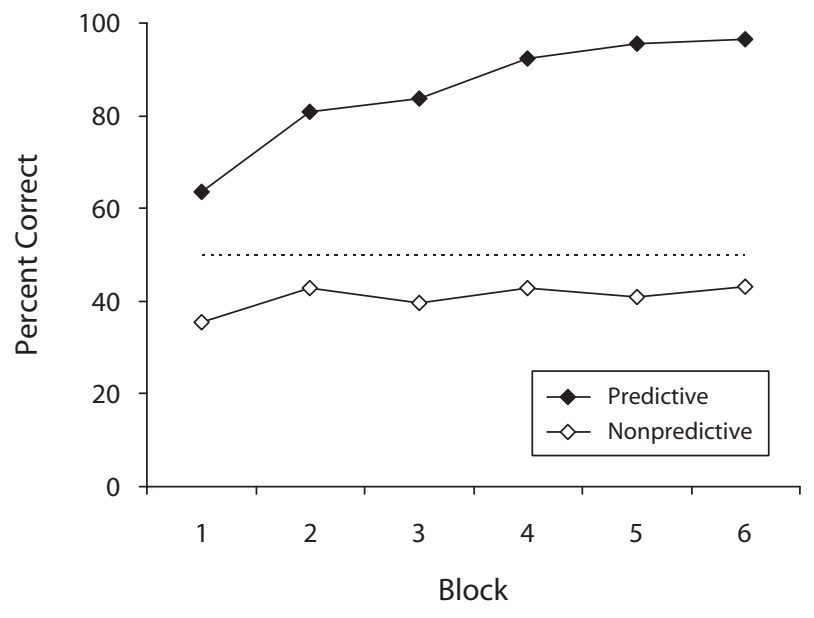

Figure 1. Mean percentages of correct responses across the six blocks of Stage 1 of Experiment 1, averaged separately for predictive and nonpredictive cues. Dotted line shows theoretical level of chance responding $(50 \%)$.

accuracy data for NP cues collapsed across Blocks 1-4 (the only blocks including data for all the participants) revealed that this trend was significant $[t(23)=4.02]$. This is almost certainly a consequence of the particular trial structure that we used. Recall that, in each block, each NP cue was paired once with each card color. Considering the sequence of outcomes paired with a particular NP cue across Stage 1, this means that the longest "run" of the same outcome paired with that cue was two. In other words, if on the previous two presentations of Cue X, the correct answer was pink, on the next presentation of $\mathrm{X}$ the correct answer must be orange. Consequently, again considering only trials involving a particular NP cue across Stage 1 (and ignoring the interceding trials, which involve other cues), alternations of outcomes were more likely than repetitions. However, if we suppose that the participants' response to Cue $\mathrm{X}$ on a particular trial was influenced by the color with which $\mathrm{X}$ was paired on its previous presentations, participants would tend to generate responses consistent with repetitions more frequently than alternations and, hence, would perform at a belowchance level. For example, if the participants experienced the previous two presentations of X paired with pink, they might be quite confident that, on its next presentation, it would again be paired with pink, and yet if they made this prediction, they would certainly be incorrect. ${ }^{2}$ We return to this issue in the introduction to Experiment 3; for the moment, it is sufficient to note that the participants clearly appreciated the difference in predictiveness of the different classes of cues during Stage 1.

Figure 2A shows the participants' mean profitability ratings for the different classes of cues across the 10 blocks of Stage 2. Preliminary analysis revealed that whether the participants received 4 or 6 blocks of Stage 1 training did not exert a significant main effect on these ratings or interact with any of the factors described below (all $p \mathrm{~s}>.1$ ), and hence, the data were collapsed over this factor in this figure and all the subsequent analyses. Learning is evident 


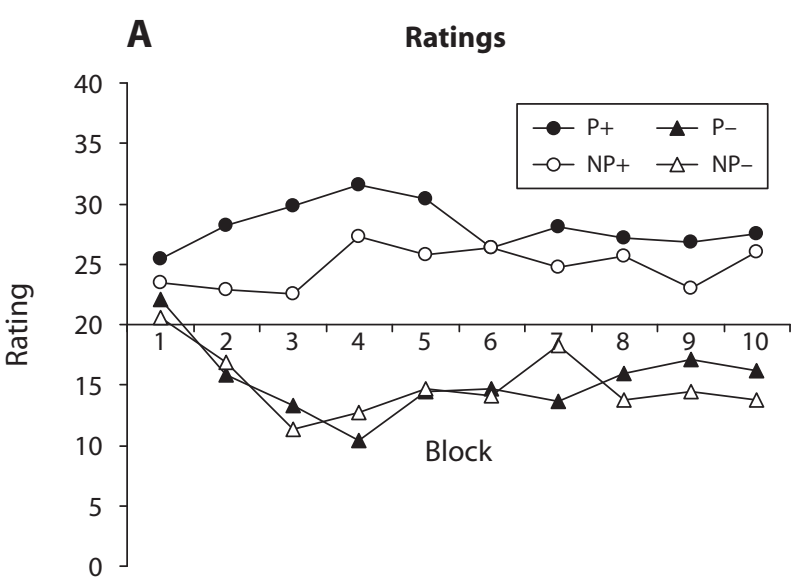

B Discrimination Scores

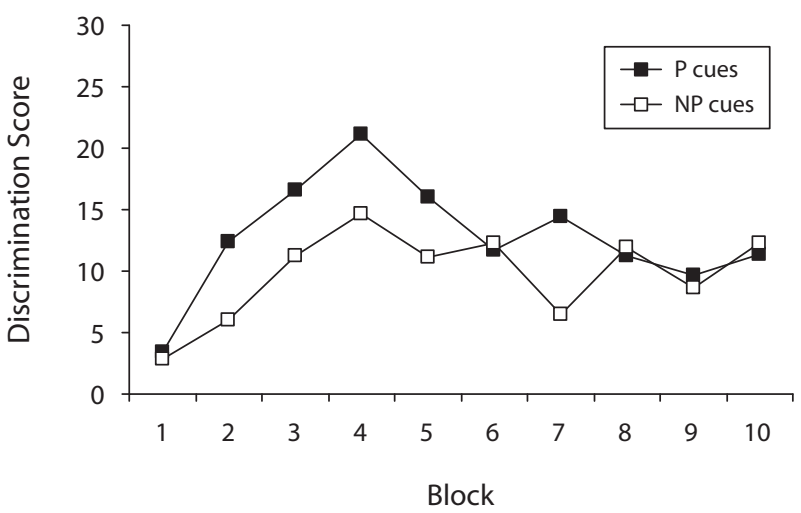

Figure 2. (A) Mean profitability ratings across the 10 blocks of Stage 2 in Experiment 1, averaged separately for previously predictive $(P)$ cues paired mainly with profit $(P+)$, previously $P$ cues paired mainly with loss $(\mathrm{P}-)$, previously nonpredictive $(\mathrm{NP})$ cues paired mainly with profit $(\mathrm{NP}+)$, and previously NP cues paired mainly with loss (NP-). High values (maximum of 40) represent the prediction that the company would make a profit; low values (minimum of 0 ) indicate the prediction of a loss. A value of 20 indicates that profit and loss were perceived as equally likely. (B) Mean discrimination scores for Stage 2 in Experiment 1, averaged separately for $P$ cues and NP cues. These scores are calculated by subtracting the mean rating received by loss-making companies from that received by profit-making companies.

from Figure 2A: The ratings for cues paired mainly with profits were consistently higher than those for cues paired mainly with losses. The reason that these learning curves do not steadily increase or decrease across training relates to the Stage 2 trial structure. Ratings for profit cues initially rose rapidly, since the first two presentations of these cues were constrained to be paired with profit. Ratings subsequently fell slightly as the participants adapted to the cue-outcome contingency that prevailed over the remaining eight presentations ( $6 / 8$ of which involved profit, and $2 / 8$ loss). Ratings for loss cues were essentially the mirror image, showing an initial rapid fall followed by a later slight rise.

The crucial question relates to the relative amount of learning about P and NP cues during Stage 2. The most appropriate measure of learning is the extent to which participants discriminate between those cues of a certain predictiveness class that predict a profit versus those that predict a loss - that is, the extent to which cues paired mainly with profits elicit higher ratings than do those paired mainly with losses. The data in Figure 2A were analyzed using an ANOVA with factors of predictiveness (note that this refers to prior predictiveness with respect to Stage 1 outcomes; all the cues were equally predictive during Stage 2), valence (paired mainly with profits vs. paired with mainly losses), and block. The data for Block 1 of Stage 2 were omitted, on the assumption that the responses during this block were guesses (but see Experiment 4). This analysis revealed a significant main effect of valence $\left[F(1,23)=45.72, M S_{\mathrm{e}}=698.75\right]$, with cues paired mainly with profits receiving higher ratings than those paired mainly with losses. The main effect of predictiveness approached significance $[F(1,23)=3.30$, $\left.M S_{\mathrm{e}}=223.53, p=.082\right]$, with $\mathrm{P}$ cues receiving marginally higher ratings than did NP cues on average. This trend implies a possible general bias toward perceiving companies paired with consistently colored business cards as more likely to make a profit than those with inconsistently colored cards, regardless of the actual valence of the outcomes with which the companies were paired. Crucially, the predictiveness $\times$ valence interaction closely approached significance $\left[F(1,23)=4.21, M S_{\mathrm{e}}=143.96\right.$, $p=.052]$; in conjunction with the pattern of means presented in Figure 2A, this indicates a trend toward better discrimination between $\mathrm{P}$ cues paired with profit or loss than for NP cues. An analysis of simple effects (which collapses over block) revealed that ratings for $\mathrm{P}$ cues paired with profit were significantly higher than those for NP cues paired with profit $\left[F(1,23)=6.33, M S_{\mathrm{e}}=23.52\right]$, but that ratings for $\mathrm{P}$ cues and NP cues paired with loss did not differ $(F<1)$. All other main effects and interactions from the ANOVA were nonsignificant (largest $F=1.59$, corresponding $p=.13$ ).

The trend toward better discrimination for $\mathrm{P}$ cues than for NP cues is shown more clearly in Figure 2B, which removes the valence factor by subtraction. That is, for each participant, a discrimination score was calculated for $\mathrm{P}$ cues by subtracting the mean rating received by loss-making $\mathrm{P}$ cues (B and $\mathrm{C}$ ) from that received by profit-making $\mathrm{P}$ cues ( $\mathrm{A}$ and $\mathrm{D})$. An equivalent score was calculated for NP cues. Figure 2B shows these mean discrimination scores across Stage 2. In further exploration of the trend toward better discrimination for $\mathrm{P}$ cues than for NP cues, mean scores for each class of cue were calculated for each participant by averaging across Blocks $2-10$ (as in the ANOVA described above). A Shapiro-Wilk test for normality (Shapiro \& Wilk, 1965) revealed that the differences in the mean discrimination score for $\mathrm{P}$ cues and the mean score for NP cues (on which the predictiveness $X$ valence interaction noted in the preceding paragraph was based) differed significantly from a normal distribution $[W(24)=.91]$. Consequently, a nonparametric test of this difference is more appropriate than the parametric analysis provided by an ANOVA. ${ }^{3}$ This revealed that the mean discrimination score across Blocks 2-10 for P cues was 
indeed significantly higher than that for NP cues [Wilcoxon's $T(24)=70]$.

The latter finding indicates better discrimination learning during Stage 2 for P cues than for NP cues. However, the results of the simple effects analysis described earlier indicate that the pattern of ratings showed an asymmetry with respect to valence: The participants gave higher ratings to $\mathrm{P}$ cues paired with profit than to NP cues paired with profit, but there was no difference for P and NP cues paired with loss. In combination with the marginally significant main effect of predictiveness, this indicates that the advantage in discrimination learning for $\mathrm{P}$ cues over NP cues interacts with a general tendency to give higher ratings to $\mathrm{P}$ cues. For cues paired with profit, the better learning about profitability for $\mathrm{P}$ cues would summate with a general tendency to give higher ratings to $\mathrm{P}$ cues, resulting in much higher ratings for $\mathrm{P}$ cues than for NP cues. For cues paired with loss, better learning that $\mathrm{P}$ cues cause a loss (which would tend to result in low ratings for these cues) would oppose a general tendency to give higher ratings to these cues, resulting in similar ratings for $P$ and NP cues.

The participants' learning during Stage 2 can also be assessed by looking at the investment choices that they made during the test stage. In analyzing these data, choice of the first item listed for each comparison in Table 1 was scored as 1 , and choice of the second item was scored as 0 . Test Types 1 and 2 ("A vs. B" and "C vs. D") both involved $\mathrm{P}$ cues paired with profit, as compared with $\mathrm{P}$ cues paired with loss, and hence were equivalent: The scores for these test types were therefore averaged and classed as "P+ vs. P-." Test Type 3 involved an NP cue paired with profit, as compared with an NP cue paired with loss, and hence was classed as "NP+ vs. NP-." Test Types 4 and 5 were equivalent (both involved $\mathrm{P}$ cues paired with profit, as compared with NP cues paired with profit) and hence were averaged and classed as " $\mathrm{P}+$ vs. NP+." Test Types 6 and 7 were equivalent ( $\mathrm{P}$ cues paired with loss, as compared with NP cues paired with loss) and hence their mean was classed as "P- vs. NP-." The resulting mean scores are shown in Figure 3; the data for Test Types 8 and 9 are not included, since these involved comparisons between equivalent cues and, hence, were uninformative (these test types were included to ensure equal exposure to all cues during the test phase). A mean score of .5 indicates chance performance, with the the participants being equally likely to choose either option.

Given a choice of "P+ vs. P-," the participants tended to choose to invest in the $\mathrm{P}$ cue that had been paired with profit $(\mathrm{P}+)$ over the $\mathrm{P}$ cue that had been paired with loss $(\mathrm{P}-)$. This tendency was assessed using a sign test ${ }^{4}$ against a hypothesized choice score of .5 (indicating chance performance), which revealed a significant difference from chance $(p<.001)$. In contrast, for the "NP+ vs. NP-" comparison, there was no significant preference for NP+ over NP- $(p=.54)$. As for the Stage 2 rating data, these indicate that discrimination learning (i.e., the extent to which the participants discriminated between cues paired with profit and those paired with loss) was greater for the $\mathrm{P}$ cues than for the NP cues, although a sign test revealed

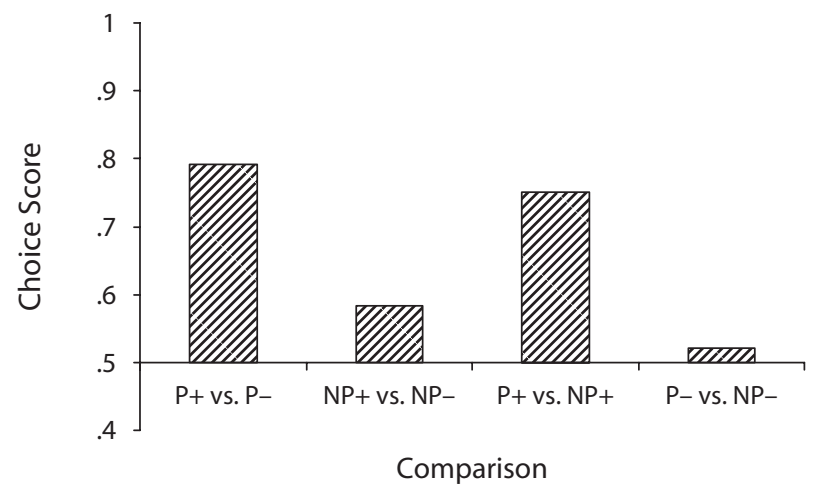

Figure 3. Mean choice scores derived from responses made during the test stage of Experiment 1. For each comparison, a choice score of 1 indicates choice of the first item listed, and a score of 0 indicates choice of the second item listed. For example, for the comparison "P+ vs. NP+," a score of 1 indicates choice of $\mathrm{P}+$, and a score of 0 indicates choice of $\mathrm{NP}+$. $\mathrm{P}$, predictive; $\mathrm{NP}$, nonpredictive.

that the tendency toward a higher choice score for " $\mathrm{P}+\mathrm{vs}$. $\mathrm{P}-$ " than for "NP+ vs. NP - " failed to reach significance $(p=.27)$.

Given a direct choice between a $\mathrm{P}+$ cue and an NP+ cue (in the "P+ vs. NP+" comparison), the participants reliably chose to invest in the $\mathrm{P}+$ cue $(p<.01)$. This could again reflect better learning about the profitability of $\mathrm{P}$ cues than about that of NP cues or simply a preference for $\mathrm{P}$ cues over NP cues in general. If the latter were true, we would expect an equally strong preference for $\mathrm{P}$ cues over NP cues in the " $\mathrm{P}-\mathrm{vs}$. NP-" comparison. If, however, this result reflects better learning about $\mathrm{P}$ cues, we would expect a preference for NP cues over $\mathrm{P}$ cues in the "P- vs. NP-" comparison: If the participants learned well that a $\mathrm{P}-$ cue predicted a loss but were less sure about an NP- cue, they should choose to invest in the NP- cue, since they would have a (relatively) greater expectation of making a profit. The results of the "P - vs. NP -" comparison provide some support for both of these accounts. There was a nonsignificant tendency for the participants to pick the $\mathrm{P}-$ cue over the $\mathrm{NP}-$ cue $(p=1.00)$. However, this tendency was significantly less strong than for the "P+vs. NP+" comparison, as revealed by a sign test comparing the two $(p<.05)$. Consequently, the advantage for $\mathrm{P}+$ cues over $\mathrm{NP}+$ cues cannot be ascribed solely to a general preference for $\mathrm{P}$ cues. Just as in the Stage 2 rating data, these findings indicate that any general preference toward P cues over NP cues interacts with significantly better discrimination learning about the profitability of $\mathrm{P}$ cues during Stage 2 . That is, in the " $\mathrm{P}+$ vs. NP+" comparison, the effect of better learning about profitability for $\mathrm{P}$ cues would summate with a general preference for $\mathrm{P}$ cues regardless of profitability, to create a strong preference for $\mathrm{P}+$ over $\mathrm{NP}+$. In the " $\mathrm{P}-\mathrm{vs}$. NP-" comparison, the effect of better learning that $\mathrm{P}-$ would result in a loss (which would tend to cause the participants to choose NP-) would counteract a general preference for $\mathrm{P}$ cues (which would tend to cause the participants to choose $\mathrm{P}-$ ), resulting in a choice score close to .5 . 
To summarize, both the rating data from Stage 2 and the choice data from the test stage indicate that the participants learned more during Stage 2 about those cues that had previously been consistent predictors of "neutral" outcomes during Stage 1 than about those cues that had been inconsistent predictors. This finding is in contrast to the prediction of Le Pelley's (2004) hybrid model of alpha effects, which anticipates that emphasizing the influence of absolute predictiveness, as compared with relative predictiveness, will tend to produce negative transfer of alpha - that is, more rapid novel learning about inconsistent predictors than about consistent predictors.

One possible reason for this discrepancy might be that the present results do not reflect the operation of an alpha process at all but, instead, derive from differences in proactive interference, with memory of Stage 1 cue-color relations interfering with learning about those same cues and profitability in Stage 2. Given the dissimilarity of the outcomes in the two stages and the fact that interference depends on the similarity between events (McGeoch \& McDonald, 1931), it seems unlikely that such interference would play a major role here. Moreover, the most natural interpretation of an interference account would suppose that $\mathrm{P}$ cues would establish strong associations during Stage 1 and, hence, be subject to more proactive interference than NP cues would be, which would form only weak associations. On this account, we would expect a greater impairment in learning about $\mathrm{P}$ than about NP cues - the opposite of the results observed.

An alternative interpretation is possible, however, in which the crucial factor is the number of sources of interference, rather than the strength of each source. P cues were paired with only one outcome in Stage 1, giving one source of interference, whereas NP cues were paired with two different outcomes, giving two sources of interference. So this alternative interpretation anticipates greater proactive interference and, hence, a greater impairment in learning for NP cues than for P cues. As such, it is able to account for our empirical findings.

To the extent that the number of sources of interference has a stronger influence than the strength of each source, then, an interference account can explain the results of Experiment 1. The aim of Experiment 2 was to test the possibility of an interference-based explanation by equating the number of sources of interference during Stage 1 for
$\mathrm{P}$ and NP cues. If interference was indeed responsible for the results of Experiment 1, we would expect this manipulation to eradicate the influence of learned predictiveness observed in that experiment.

\section{EXPERIMENT 2}

Table 2 shows the design of Experiment 2. Stage 1A was the game as in Experiment 1 and comprised six blocks. In Stage 1B, which also comprised six blocks, all outcomes changed: Where a particular cue had been paired with pink in Stage 1A, it was paired with orange in Stage 1B, and vice versa. This ensured that across Stages $1 \mathrm{~A}$ and $1 \mathrm{~B}$, all the cues were paired with each outcome exactly six times and, hence, the number of sources of interference was equal for all the cues. The only difference between cues was in how these pairings were distributed across training. We will continue to refer to Cues A-D as P cues, since these cues were predictive in each of Stages 1A and 1B considered separately (although nonpredictive when considered over the whole of Stage 1; this raises certain issues with regard to how predictiveness is characterized, which are taken up in the Results and Discussion section below). In contrast, Cues $\mathrm{X}$ and $\mathrm{Y}$ were nonpredictive in each stage considered separately and across Stage 1 as a whole and, hence, will be referred to as NP cues.

The only other procedural difference, as compared with Experiment 1, related to the pattern of Stage 2 outcomes. Experiment 1 used a majority pattern in Stage 2, where cues were paired with eight outcomes of one valence and two of the opposite valence. This manipulation was intended to slow Stage 2 learning in order to prevent ceiling effects from masking any differences in learning. In the event, however, Stage 2 learning proceeded rather too slowly, with ratings never deviating far from the midpoint of the scale (see Figure 2), and the pattern of these ratings was noisy. Consequently, Experiment 2 used a consistent pattern of outcomes across Stage 2: The cues were paired with either all profits or all losses.

\footnotetext{
Method

Participants and Stimuli. Twenty-nine Cardiff University students participated in exchange for either $£ 5$ or course credit. The data from 2 of these participants were subsequently excluded on the basis of the criterion used in Experiment 1. The stimuli were the same as those in Experiment 1.
}

Iable 2

Design of Experiment 2

\begin{tabular}{|c|c|c|c|c|c|}
\hline \multicolumn{2}{|c|}{ Stage $1 \mathrm{~A}$} & \multicolumn{2}{|c|}{ Stage 1B } & \multirow{2}{*}{$\frac{\text { Stage } 2}{\mathrm{~A}+}$} & \multirow{2}{*}{$\frac{\text { Test }}{\text { 1. A vs. B }}$} \\
\hline $\mathrm{A} \rightarrow$ Pink & $\mathrm{A} \rightarrow$ Pink & $\mathrm{A} \rightarrow$ Orange & $\mathrm{A} \rightarrow$ Orange & & \\
\hline $\mathrm{B} \rightarrow$ Orange & $\mathrm{B} \rightarrow$ Orange & $\mathrm{B} \rightarrow$ Pink & $\mathrm{B} \rightarrow$ Pink & $\mathrm{B}+$ & $\begin{array}{l}\text { 2. C vs. D } \\
\text { 3. X vs. Y }\end{array}$ \\
\hline $\mathrm{C} \rightarrow$ Orange & $\mathrm{C} \rightarrow$ Orange & $\mathrm{C} \rightarrow$ Pink & $\mathrm{C} \rightarrow$ Pink & $\mathrm{C}+$ & $\begin{array}{l}\text { 4. A vs. X } \\
\text { 5. C vs. X }\end{array}$ \\
\hline $\mathrm{D} \rightarrow$ Pink & $\mathrm{D} \rightarrow$ Pink & $\mathrm{D} \rightarrow$ Orange & $\mathrm{D} \rightarrow$ Orange & $\mathrm{D}-$ & 6. B vs. Y \\
\hline $\mathrm{X} \rightarrow$ Pink & $\mathrm{X} \rightarrow$ Orange & $\mathrm{X} \rightarrow$ Pink & $\mathrm{X} \rightarrow$ Orange & $\mathrm{X}+$ & $\begin{array}{l}\text { 7. D vs. Y } \\
\text { 8. A vs. C }\end{array}$ \\
\hline $\mathrm{Y} \rightarrow$ Pink & $\mathrm{Y} \rightarrow$ Orange & $\mathrm{Y} \rightarrow$ Pink & $\mathrm{Y} \rightarrow$ Orange & $\mathrm{Y}+$ & 9. B vs. D \\
\hline
\end{tabular}




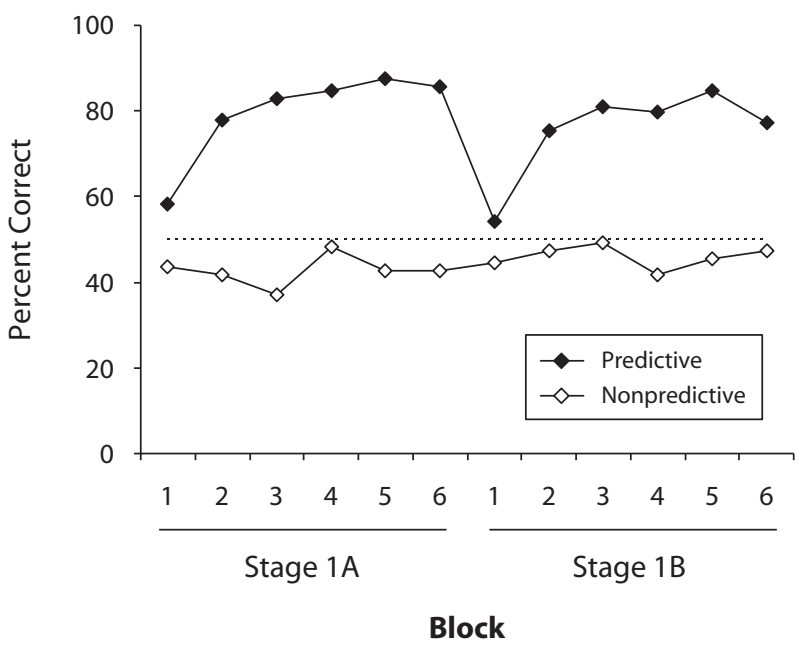

Figure 4. Mean percentages of correct responses across Stages 1A and 1B of Experiment 2, averaged separately for predictive cues and nonpredictive cues. The dotted line shows the theoretical level of chance responding $(50 \%)$.

Procedure. Stage 1B followed immediately from Stage 1A with no break or explicit indication to the participants that the cue-outcome relationships had changed. Stages 1A and 1B both comprised six training blocks. The outcomes paired with each cue were consistent across Stage 2; cues shown with the "+" symbol in Table 2 were paired with profit on all of their 10 presentations, and cues shown with "-" were paired with loss on all 10 presentations. Other details were the same as in Experiment 1.

\section{Results and Discussion}

Figure 4 shows mean percentages of correct responses per block across Stages 1A and 1B. For the P cues, accuracy increased across Stage 1A before falling sharply in Block 1 of Stage 1B. Given that responses to P cues learned in Stage 1A were incorrect in Stage 1B, we might expect performance with these cues in Block 1 to fall below chance, and yet it remained above $50 \%$ [albeit nonsignificantly; $t(26)=1.12, p=.27]$. This presumably was a result of within-block learning. Each P cue appeared twice during each Stage 1 block, and any learning about the changed cue-outcome relationship on its first presentation would tend to lead to above-chance performance on the second, thus ameliorating the effect of the unexpected change in outcomes. As in Experiment 1, accuracy of responses to NP cues remained below 50\% throughout Stages 1A and 1B; collapsing across the six blocks of each stage reveals that this trend was significant in Stage 1A $[t(26)=4]$ and approached significance in Stage 1B $[t(26)=2, p=.056]$.

Figure $5 \mathrm{~A}$ shows mean profitability ratings for the different classes of cues in Stage 2. An ANOVA with factors of predictiveness, valence, and block (omitting Block 1 data, as in Experiment 1) revealed a significant main effect of valence $\left[F(1,26)=118.84, M S_{\mathrm{e}}=818.73\right]$. The main effect of predictiveness did not reach significance $\left[F(1,26)=2.07, M S_{\mathrm{e}}=232.22, p=.16\right]$, although there was a numerical trend toward higher ratings for $\mathrm{P}$ cues than for NP cues, as in Experiment 1. The block $\times$ valence interaction was significant $\left[F(8,208)=11.96, M S_{\mathrm{e}}=\right.$ 91.63], with Figure 5A showing that discrimination between cues paired with profits and those paired with losses improved across the course of training. Crucially, there was a significant interaction between predictiveness and valence $\left[F(1,26)=5.91, M S_{\mathrm{e}}=167.60\right]$; given the pattern of means in Figure 5A, this interaction indicates that there was significantly better discrimination between $P$ cues paired with profit or loss than between NP cues. A simple effects analysis revealed that ratings for $P$ cues paired with profit were significantly higher than those for NP cues paired with profit $\left[F(1,26)=6.39, M S_{\mathrm{e}}=24.77\right]$ but that ratings for $\mathrm{P}$ cues and NP cues paired with loss did not differ $(F<1)$. Hence, the pattern of ratings showed an asymmetry with regard to valence similar to that observed in Experiment 1. All other main effects and interactions were nonsignificant (largest $F=1.23$, corresponding $p=$ .28). Figure 5B shows discrimination scores for these cues (calculated as for Experiment 1); the advantage for $\mathrm{P}$ cues over NP cues is clear. A paired $t$ test revealed that the mean discrimination score across Blocks 2-10 for $\mathrm{P}$ cues was significantly higher than that for NP cues $[t(26)=2.43]$.

Figure 6 shows mean choice scores for the various comparisons assessed in the test phase, calculated as for Experiment 1. Given a choice of "P+ vs. P-," the partici-
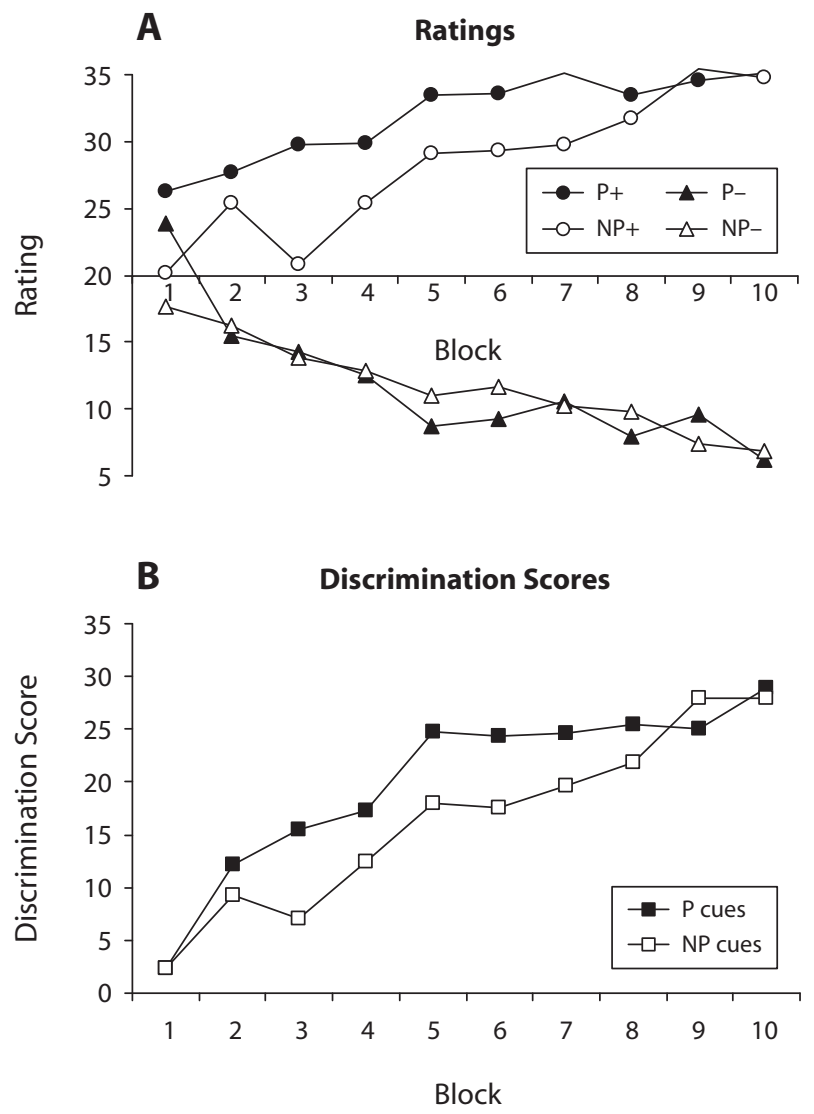

Figure 5. (A) Mean profitability ratings for Stage 2 in Experiment 2, averaged as in Figure 2A. (B) Mean discrimination scores for Stage 2 of Experiment 2, calculated and averaged as in Figure $2 B$. P, predictive; NP, nonpredictive. 


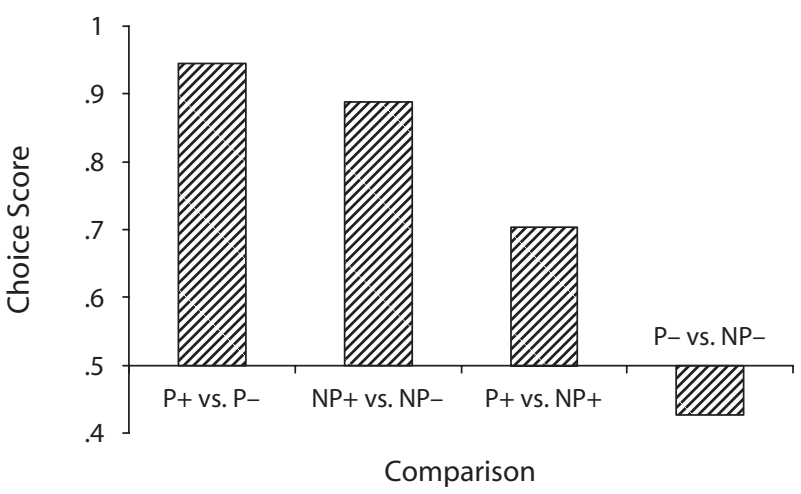

Figure 6. Mean choice scores derived from responses made during the test stage of Experiment 2, calculated as in Figure 3. P, predictive; NP, nonpredictive.

pants were significantly more likely to choose to invest in $\mathrm{P}+$ rather than in $\mathrm{P}-($ sign test $p<.001)$. Likewise, given a choice of "NP+ vs. NP-," the participants were significantly more likely to choose NP+over NP- $(p<$ $.001)$. Although the preference for cues paired with profit over those paired with loss was numerically greater for $\mathrm{P}$ cues than for NP cues (consistent with the idea of better learning about the profitability of $\mathrm{P}$ cues), this difference failed to reach significance $(p=1)$. This is unsurprising; the consistency of outcomes throughout Stage 2 meant that Stage 2 learning about profitability was stronger in Experiment 2 than in Experiment 1 and, hence, straight choices between profit-making and loss-making cues were likely to be near ceiling.

As for Experiment 1, given a choice of "P+ vs. NP+," the participants reliably chose to invest in the $\mathrm{P}+$ cue $(p<.05)$. This cannot reflect merely a general preference for $\mathrm{P}$ cues, since the same pattern was not observed in the "P- vs. NP-" comparison, where the numerical (but nonsignificant) preference was for the $\mathrm{NP}-$ cue ( $p=$ .48). Crucially, the mean choice scores were significantly different for the "P+ vs. NP+" and "P - vs. NP-" comparisons $(p<.05)$. This is the result that would be anticipated if the participants had learned more about the profitability of $\mathrm{P}$ cues than about that of NP cues, which would lead them to choose $\mathrm{P}+$ over $\mathrm{NP}+$ and $\mathrm{NP}-$ over $\mathrm{P}-$. The fact that the choice scores for these comparisons are not symmetrical around .5 indicates that this greater discrimination learning about $\mathrm{P}$ cues interacts with a general preference for $\mathrm{P}$ cues regardless of their profitability, as in Experiment 1.

Experiment 2 once again demonstrated (in both rating data and choice data) better discrimination learning about $P$ cues than about NP cues, despite equating the number of sources of proactive interference for each, which implies that proactive interference is not the source of this influence of learned predictiveness. These results therefore support an analysis of this learned predictiveness effect in terms of changes in the alpha of cues, with alpha determined by experienced predictive history. Thus, it would seem that in human contingency learning, prior experience of cues as predictive allows those cues to maintain a higher alpha than do cues experienced as nonpredictive, regardless of whether pretraining is with individual cues or with multiple simultaneously presented cues.

The finding of a Stage 2 advantage for $\mathrm{P}$ cues, despite the fact that all the cues were nonpredictive when assessed over the whole of Stage 1, places constraints on how this alpha mechanism should be characterized. Specifically, it indicates that predictiveness is not assessed by taking into account all of our experience with a cue. That is, when considering Cue A, people do not bring to mind all prior instances involving Cue A and then calculate the alpha of A on the basis of the consistency of the outcomes with which it has been paired over this whole set (cf. De Houwer, Beckers, \& Vandorpe, 2005), because, on such an account, all the cues in Experiment 2 would be equivalent. Instead, the results fit well with an associative view, in which alpha is updated on a trial-by-trial basis, because associative models typically instantiate a recency bias, wherein recent information has a greater effect on the current state of the model than does temporally distant information (see López, Shanks, Almaraz, \& Fernández, 1998). Applying this idea to alpha, if a cue has been predictive over its most recent presentations, the alpha of that cue will be higher than if it has not been predictive over its recent presentations. This will ensure, that at the outset of Stage 2, Cues A-D (which have been consistently paired with the same outcome on their six most recent presentations) will have a higher alpha than will Cues $\mathrm{X}$ and $\mathrm{Y}$ (which have been nonpredictive throughout Stage 1). We note, however, that these results do not prove an associative basis to alpha. For example, they are also consistent with an inferential account in which the impact of alpha processes on contingency judgments is mediated by conscious propositional knowledge (De Houwer et al., 2005), as long as these alpha processes are biased toward more recent information. Such an account is essentially a redescription of the associative model of these effects.

\section{EXPERIMENT 3}

The present experiments were motivated by the suggestion that pretraining with individual cues (which are the best available predictors of reinforcement) might produce different learned predictiveness effects than does pretraining with compounds of multiple cues (some of which are better predictors of reinforcement than are others), as a consequence of differences in the extent to which relative predictiveness mechanisms (e.g., Mackintosh, 1975) apply to these different situations. Specifically, individual-cue pretraining should minimize the contribution of relative predictiveness mechanisms. Experiments 1 and 2, however, showed learned predictiveness effects in humans following single-cue pretraining that are similar to those reported following pretraining with multiple cues; in all cases, better learning was observed for cues previously established as predictive than for those established as nonpredictive.

Before concluding that absolute predictiveness mechanisms produce positive transfer of alpha in humans, however, we must consider an important conceptual difference 
between Experiments 1 and 2 and the analogous animal study by Wilson et al. (1992). As was noted earlier, in Wilson et al.'s experiments, the individually pretrained light was the best available predictor of the tone in all groups (albeit an imperfect predictor in Group Experimental, for which it followed $50 \%$ of the presentations of the light). It could be argued, however, that in the present Experiments 1 and 2, the NP cues were not the best available predictors of Stage 1 outcomes. Recall that the participants' prediction accuracy for these cues was consistently and significantly below chance during Stage 1, as a consequence of the particular trial structure used. As a result, each NP cue was actually a poorer predictor of Stage 1 outcomes than was the experimental context, which predicted neither outcome more than the other and, hence, would give chance performance. In other words, on presentation of an NP cue, the participants would have performed better by ignoring that cue and guessing randomly than by basing their prediction on their experience of the cue. This raises the possibility that the individual-cue pretraining procedure of Experiments 1 and 2 might not have eradicated the influence of relative predictiveness mechanisms, an argument that cannot be applied to the animal studies. Hence the impairment in learning for NP cues in Experiments 1 and 2 might reflect the operation of relative predictiveness mechanisms, just as in studies employing multiple-cue pretraining (e.g., Le Pelley \& McLaren, 2003).

This possibility was tested in Experiment 3, which used a design (Table 3 ) in which all the cues were, without doubt, the best available predictors of the outcomes occurring during Stage 1. On each Stage 1 trial, the participants had to predict which of three different colors of business card was used by a particular company. Given that the manipulation used in Experiment 2 to equate the number of sources of interference seemed to have little effect on the pattern of results observed (both Experiments 1 and 2 demonstrated better learning about the profitability of $\mathrm{P}$ cues than about that of NP cues), for simplicity this manipulation was not used in Experiment 3. Cues A-F were consistently paired with a single color throughout Stage 1 and, hence, are labeled $P$ cues. In contrast, each of Cues $\mathrm{U}-\mathrm{Z}$ was paired with two of the three available colors on different trials. For example, in each block, Cue U would be paired once with blue and once with green, but

Iable 3

Design of Experiment 3

\begin{tabular}{|c|c|c|c|c|}
\hline \multicolumn{3}{|c|}{ Stage 1} & Stage 2 & Test \\
\hline$P$ cues & $\begin{array}{l}\mathrm{A} \rightarrow \text { Blue } \\
\mathrm{B} \rightarrow \text { Green } \\
\mathrm{C} \rightarrow \text { Yellow } \\
\mathrm{D} \rightarrow \text { Blue } \\
\mathrm{E} \rightarrow \text { Green } \\
\mathrm{F} \rightarrow \text { Yellow }\end{array}$ & $\begin{array}{l}\mathrm{A} \rightarrow \text { Blue } \\
\mathrm{B} \rightarrow \text { Green } \\
\mathrm{C} \rightarrow \text { Yellow } \\
\mathrm{D} \rightarrow \text { Blue } \\
\mathrm{E} \rightarrow \text { Green } \\
\mathrm{F} \rightarrow \text { Yellow }\end{array}$ & $\begin{array}{l}\mathrm{A}+ \\
\mathrm{B}+ \\
\mathrm{C}+ \\
\mathrm{D}- \\
\mathrm{E}- \\
\mathrm{F}-\end{array}$ & $\begin{array}{l}\text { 1. A vs. D } \\
\text { 2. B vs. E } \\
\text { 3. C vs. F } \\
\text { 4. U vs. X } \\
\text { 5. V vs. Y } \\
\text { 6. W vs. Z }\end{array}$ \\
\hline NP cues & $\begin{array}{l}\mathrm{U} \rightarrow \text { Blue } \\
\mathrm{V} \rightarrow \text { Green } \\
\mathrm{W} \rightarrow \text { Yellow } \\
\mathrm{X} \rightarrow \text { Blue } \\
\mathrm{Y} \rightarrow \text { Green } \\
\mathrm{Z} \rightarrow \text { Yellow }\end{array}$ & $\begin{array}{l}\mathrm{U} \rightarrow \text { Green } \\
\mathrm{V} \rightarrow \text { Yellow } \\
\mathrm{W} \rightarrow \text { Blue } \\
\mathrm{X} \rightarrow \text { Green } \\
\mathrm{Y} \rightarrow \text { Yellow } \\
\mathrm{Z} \rightarrow \text { Blue }\end{array}$ & $\begin{array}{l}\mathrm{U}+ \\
\mathrm{V}+ \\
\mathrm{W}+ \\
\mathrm{X}- \\
\mathrm{Y}- \\
\mathrm{Z}-\end{array}$ & $\begin{array}{l}\text { 7. A vs. U } \\
\text { 8. B vs. V } \\
\text { 9. C vs. W } \\
\text { 10. D vs. X } \\
\text { 11. E vs. Y } \\
\text { 12. F vs. Z }\end{array}$ \\
\hline
\end{tabular}

never with yellow. Consequently, these cues were inconsistent predictors and, hence, are labeled NP cues. However, these NP cues were better predictors of the outcome with which they were paired than was the experimental context, which was paired with all three outcomes equally. If, on trials involving an NP cue, participants were to base their prediction on the context (or a random guess), they would be equally likely to choose any of the three colors. If, however, they were to attend to the presented cue and base their prediction on their prior experience with that cue, then they would tend to select one of the two appropriate colors (e.g., blue or green if Cue $\mathrm{U}$ was presented) and avoid the other, inappropriate color. Thus, the NP cues do not predict which specific outcome will occur on a given trial but do predict which specific outcome will not occur and, in this sense, are better predictors than is the context. By looking at the participants' choices of appropriate versus inappropriate outcomes during Stage 1, we can verify that the participants indeed based their predictions on the identities of the NP cues, rather than ignoring these cues in favor of the context.

Stage 2 was the same as in Experiments 1 and 2: P cues and NP cues were paired with either profit or loss, and learning was assessed by means of ratings provided on each trial and in a final test phase in which the participants chose which of two companies to invest in on each trial.

\section{Method}

Participants and Stimuli. Thirty-nine Cardiff University students participated in exchange for course credit. The data from 5 of these participants were subsequently excluded on the basis of the criterion used in Experiment 1. The company names were Sparkly Sheep, G. A. Davies, Powell's, Mary Humphries, Big Bear, Gee, Lollie, Not So Big Kids, EKPK, Pomona, Brass Pig, and ZeBra. Other details were the same as in Experiment 1.

Procedure. On each Stage 1 trial, three business cards were shown laid out in a triangle and differing only in background color: one blue, one green, and one yellow. The order in which the three colors were laid out was determined randomly for each participant but remained constant across Stage 1 training. The participants experienced eight blocks of Stage 1 training; other procedural details were the same as in Experiment 1. Stage 2 comprised six blocks, with consistent outcomes across all blocks, as in Experiment 2. Finally, the test phase presented each of the choices shown in Table 3 and followed the procedure in Experiment 1.

\section{Results and Discussion}

Figure 7A shows mean percentages of correct responses per block across Stage 1. A one-sample $t$ test revealed that, across the latter half of Stage 1, the participants' accuracy for NP cues was significantly greater than the chance level of $33.3 \%[t(33)=2.62]$. This demonstrates that the participants had indeed learned that the NP cues were partially predictive. However, performance with these cues was certainly not far above chance: If the participants had learned perfectly that each NP cue could be paired with only two of the three possible outcomes, accuracy could potentially have risen to $50 \%$, because each time an NP cue was presented, one of the outcome options could be ruled out. Accuracy for the NP cues never approached this $50 \%$ level, however. One possible explanation is that the participants learned the partial predictiveness of NP cues 

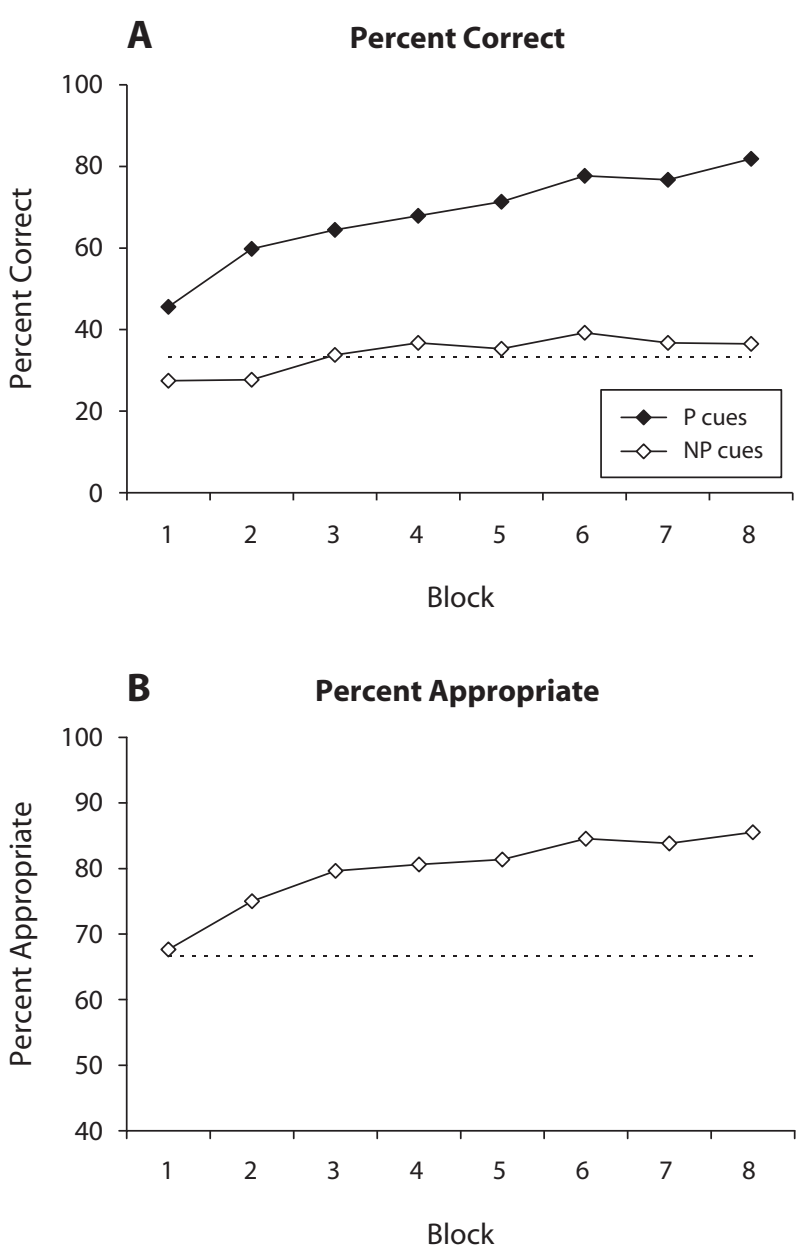

Figure 7. (A) Mean percentages of correct responses across Stage 1 in Experiment 3, averaged separately for predictive (P) cues and nonpredictive (NP) cues. The dotted line shows the theoretical level of chance responding $(33.3 \%)$. (B) Mean percentages of appropriate responses to NP cues across Stage 1 in Experiment 3, where a response was deemed appropriate if the participant chose an outcome that could feasibly be paired with that NP cue.

poorly; that is, they were slow to acquire the knowledge that each NP cue could be paired with only two of the three possible outcomes. An alternative possibility is that the participants acquired this knowledge rapidly but that the influence of the particular trial structure used in these experiments (which favored alternations of outcomes, rather than repetitions: see the Results and Discussion section in Experiment 1) might have led to accuracy remaining below 50\%, much as it did for NP cues in Experiments 1 and 2 .

Evidence supporting the latter suggestion comes from an analysis of participants' particular choices on trials involving NP cues. Figure 7B shows the percentage of appropriate responses made to NP cues during Stage 1. A response was deemed appropriate if the participant chose an outcome that could feasibly be paired with that NP cue. For example, on a trial involving Cue $\mathrm{U}$, a choice of either blue or green was appropriate (since U was paired with blue and green during Stage 1), but a choice of yellow was inappropriate (since $U$ was never paired with yellow). Chance responding corresponded to a mean of $66.7 \%$ appropriate responses. The participants' scores rapidly rose above chance: A simple effects analysis demonstrated that appropriate responding to NP cues was significantly greater than chance from Block 2 onward [smallest $F(1,33)=13.05$, corresponding $p<.01]$.

These data clearly demonstrate that the participants learned about the partial predictiveness of NP cues. Moreover, we can be confident that on trials involving $\mathrm{NP}$ cues, the participants attended to the presented cue and responded on the basis of the identity of that cue, rather than basing their prediction on the context (or a random guess).

Figure $8 \mathrm{~A}$ shows mean profitability ratings across Stage 2, which were very similar to the corresponding data in Experiment 2. An ANOVA with factors of predictiveness, valence, and block (omitting Block 1 data) revealed a significant main effect of valence $[F(1,33)=68.35$, $\left.M S_{\mathrm{e}}=710.47\right]$. Discrimination between cues paired with profits and those paired with losses improved across Stage 2 training, and the block $\times$ valence interaction demonstrates that this trend was significant $[F(4,132)=$

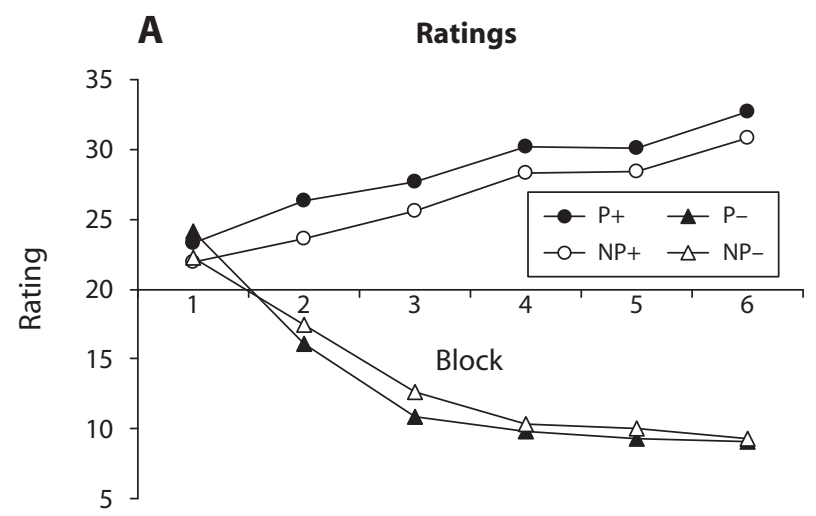

B Discrimination Scores

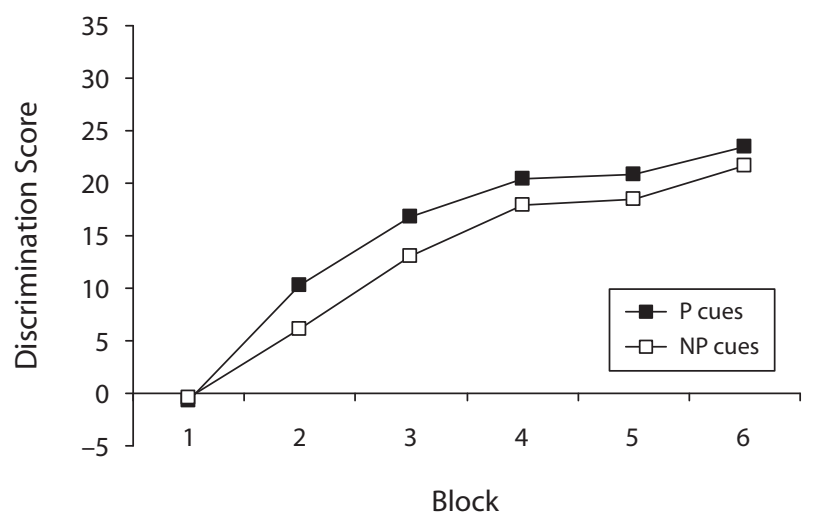

Figure 8. (A) Mean profitability ratings across Stage 2 in Experiment 3, averaged as in Figure 2A. (B) Mean discrimination scores for Stage 2 in Experiment 3, calculated and averaged as in Figure 2B. P, predictive; NP, nonpredictive. 
$\left.21.84, M S_{\mathrm{e}}=48.57\right]$. Crucially, there was a significant interaction between predictiveness and valence $[F(1,33)=$ 4.91, $\left.M S_{\mathrm{e}}=74.57\right]$; given the pattern of means in Figure $8 \mathrm{~A}$, this interaction indicates that there was significantly better discrimination between $\mathrm{P}$ cues paired with profit or loss than between NP cues. A simple effects analysis revealed that ratings for $\mathrm{P}$ cues paired with profit were significantly higher than ratings for NP cues paired with profit $\left[F(1,23)=6.33, M S_{\mathrm{e}}=23.52\right]$ but that ratings for $P$ cues and NP cues paired with loss did not differ $(F<1)$. Hence, the pattern of ratings showed an asymmetry with regard to valence similar to that observed in Experiments 1 and 2. All other main effects and interactions were nonsignificant (largest $F=1.51$, corresponding $p=$ .20). Figure 8B shows discrimination scores for these cues (calculated as in Experiment 1); the advantage for $\mathrm{P}$ cues over NP cues is clear. A paired $t$ test revealed that the mean discrimination score across Blocks 2-6 for $\mathrm{P}$ cues was significantly higher than that for NP cues $[t(33)=2.22]$. As in the previous experiments, then, discrimination learning was greater for $\mathrm{P}$ cues than for NP cues.

Figure 9 shows mean choice scores for the comparisons assessed in the test phase. As in Experiments 1 and 2, choice of the first item listed for each comparison in Table 3 was scored as 1 , and choice of the second item as 0 . Test Types 1-3 were averaged as type " $\mathrm{P}+$ vs. $\mathrm{P}-$," Types 4-6 were averaged as type "NP+ vs. NP-," Types 7-9 were averaged as type "P+ vs. NP+," and Types 10-12 were averaged as type " $\mathrm{P}-\mathrm{vs.} \mathrm{NP}-$."

Given a choice of "P+ vs. $\mathrm{P}-$," the participants were significantly more likely to choose to invest in $\mathrm{P}+$ than in $\mathrm{P}-($ sign test $p<.001)$. Likewise, given a choice of "NP+ vs. NP-," the participants reliably chose NP+ over NP$(p<.001)$. Although the preference for cues paired with profit over those paired with loss was numerically greater for $\mathrm{P}$ cues than for NP cues (consistent with better learning about the profitability of $\mathrm{P}$ cues), as in Experiment 2, this difference failed to reach significance $(p=.39)$. And although there was a trend toward higher choice scores for the "P+ vs. NP+" comparison, as compared with the "P - vs. NP-" comparison (as in previous experiments), this difference was nonsignificant ( $p=.17$ ), and neither

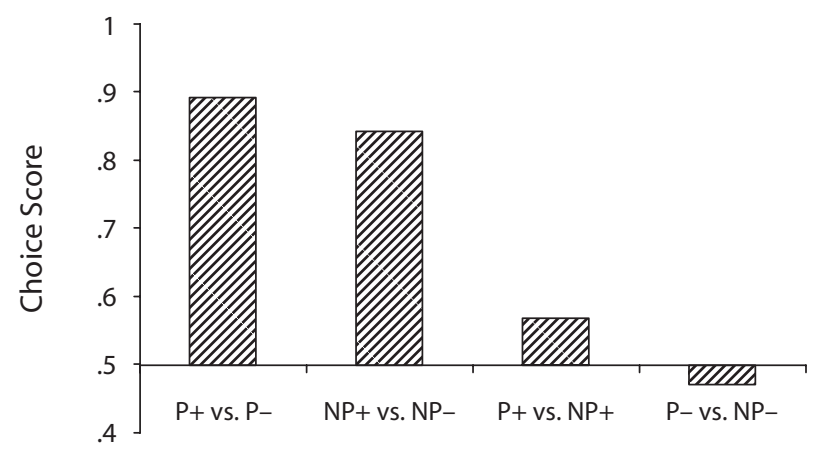

Comparison

Figure 9. Mean choice scores derived from responses made during the test stage in Experiment 3, calculated as in Figure 3. P, predictive; NP, nonpredictive. of the scores for these comparisons differed significantly from chance ( $p=.12$ and $p=.86$, respectively).

Experiment 3 once again demonstrated better discrimination learning about $\mathrm{P}$ cues than about NP cues in Stage 2 ratings. Given the analysis of the participants' choices on Stage 1 trials involving NP cues, we can be confident that they indeed attended to these cues and responded on the basis of their identity throughout Stage 1. This makes it unlikely that relative predictiveness mechanisms were the source of this advantage for $\mathrm{P}$ cues, since for both P and NP cues, the participants clearly learned that the presented cue was the best available predictor of the outcome on each trial. Instead, it would seem that the advantage for $\mathrm{P}$ cues derived from their greater absolute predictiveness.

No significant effect of prior predictiveness was observed in the choice data from the test phase in Experiment 3, although all the trends were in the same direction as in the previous experiments. This presumably reflects the lower sensitivity of the choice test, as compared with the analysis of rating data. The choice test comes only after Stage 2 training is complete, by which time learning may be approaching asymptote for both classes of cue, and, moreover, produces categorical data that limit the power of statistical analysis.

\section{EXPERIMENT 4}

In the Stage 2 rating data of each of Experiments 1-3, we observed the critical interaction between predictiveness and valence. That is, participants' ratings discriminated more strongly between $P$ cues that were paired with different outcomes than between NP cues that were paired with different outcomes. We have taken this interaction as evidence for better discrimination learning regarding $P$ cues than regarding NP cues.

However, another aspect of our findings thus far might give us pause when deciding whether to accept this conclusion. In each of Experiments 1-3, there was at least a hint of asymmetry in these ratings. We have argued that this asymmetry likely reflects a general bias, wherein participants perceive companies previously paired with consistently colored business cards as being more likely to make a profit than companies paired with inconsistently colored cards.

As an example, we argued earlier that the participants' ratings of companies during the first block of Stage 2 reflected guesses. This would be true to the extent that there was not an opportunity for feedback-driven learning about the consequences of these cues during this first block. But it is certainly possible that the more general bias described in the preceding paragraph exerted a systematic effect on the participants' ratings during this first block (indeed, one might expect the effect of this bias to be greatest in Block 1, before it was counteracted by any specific learning about the relationships between cues and profitability outcomes). And indeed, across Experiments 1-3, profitability ratings for $\mathrm{P}$ cues were, on average, higher than those for NP cues during the first block of Stage 2; the relevant data are shown in Figures 2A, 5A, and 8A. This trend was highly 
significant in Experiment $2[t(26)=3.94]$ but failed to reach significance in Experiments 1 and $3[t(23)=1.06$, $p=.30$, and $t(33)=1.38, p=.18$, respectively].

The likely reason for this bias is that the outcomes used in Stage 2 (profit and loss) were evaluatively distinct; a profit is a positive outcome, and a loss is a negative outcome. It seems plausible that the participants might have felt more positively toward those cues that were predictive during Stage 1 than toward those cues that were nonpredictive. This might be because they believed that companies that produced consistently colored business cards would be more organized and efficient and, hence, more likely to make a profit. Alternatively, the participants might simply have liked these company names more, because, by the end of Stage 1, they knew the correct color responses to make for these companies (as compared with the NP cues, which were always associated with a mixture of correct and incorrect responses). Whatever the reason, it follows naturally that the participants would have seen companies about which they felt more positively as being more likely to be associated with a positive outcome (profit) at the outset of Stage 2, when they had no other information on which to base their decisions.

It is because of this possibility of a bias toward seeing $\mathrm{P}$ cues as generally more likely to make a profit that we have focused on discrimination between cues paired with profit and those paired with loss as our index of learning. That is, although ratings might be higher for $\mathrm{P}$ cues than for NP cues in general, higher ratings for $\mathrm{P}$ cues paired with profit than for $\mathrm{P}$ cues paired with loss must reflect learning about specific cue-outcome relationships, and the greater this difference, the more learning there has been - that is, the better participants have learned to discriminate between these cues. Consequently, we have argued that the significant predictiveness $\times$ valence interaction found in Experiments 1-3 indicates better learning about $\mathrm{P}$ cues than about NP cues.

Nevertheless, it is arguably possible for an account based on a general bias toward $\mathrm{P}$ cues, rather than a difference in rate of learning about $\mathrm{P}$ cues and NP cues, to explain the emergence of better discrimination between $P$ cues than between NP cues as Stage 2 proceeds. The details of this account are rather complicated and are omitted here. This possibility, however, provided the motivation to demonstrate a discrimination learning advantage for $\mathrm{P}$ cues over NP cues in a situation in which any general bias toward $\mathrm{P}$ cues would be unlikely to exert an influence. The simplest way to do this was to use Stage 2 outcomes that were not evaluatively distinct - that is, outcomes where one was not intrinsically "better" or "worse" than the other. This was the approach taken in Experiment 4, which used exactly the same design as Experiment 3 (see Table 3), but with outcomes in Stage 2 that were intended to be evaluatively equivalent.

\section{Method}

Participants and Stimuli. Twenty-one Cardiff University students participated in exchange for payment of $£ 6$. No participants' data were excluded on the basis of the criterion used in Experiment 1 . Company names were the same as those in Experiment 3.
Procedure. Stage 1 training was exactly the same as that in Experiment 3. Following Stage 1, instructions informed the participants that on each trial, they would be told that they had visited the cafeteria of one of the companies and would be asked to predict whether "fish and chips" or "burger and chips" would be the cafeteria's best-selling meal on the day that they visited. Stage 2 training was the same as that in Experiment 3, but the labeling of the rating scale was changed: The label "Sure to be fish and chips" was displayed at one pole, the label "Sure to be burger and chips" at the other, and the label "Both meals equally likely" in the center. The orientation of the scale was counterbalanced across participants; for 11 participants, "Sure to be fish and chips" was at the left-hand pole, whereas for the remaining 10 participants, it was at the righthand pole. As in the previous experiments, immediate feedback was provided once the participants had made their ratings; the message "The best-selling meal was:" followed by the name of the appropriate meal appeared, written in blue. For companies shown as paired with outcome "+" in Table 3, the correct answer during Stage 2 was "fish and chips"; for companies shown as paired with outcome "-", the correct answer was "burger and chips."

Experiment 4 also included a final choice test that was similar to that in Experiment 3. As in Experiment 3, the results of this choice test were inconclusive. In each critical comparison, there was a numerical trend in the direction that would suggest better Stage 2 learning about $\mathrm{P}$ cues than about NP cues, but, again as in Experiment 3, these trends were nonsignificant (all $p \mathrm{~s}>.2$ ). Consequently, for the sake of brevity, further discussion of this choice test and the results arising from it is omitted (full details are available from the first author on request).

\section{Results and Discussion}

Data from Stage 1 training were very similar to those in Experiment 3 (which is unsurprising, given that the same training procedure was used in each case). Figure 10A shows mean percentages of correct responses per block across Stage 1. A one-sample $t$ test revealed that, across the latter half of Stage 1, participants' accuracy for NP cues was significantly greater than the chance level of $33.3 \%[t(20)=5.36]$. Figure 10B shows the percentage of appropriate responses made to NP cues during Stage 1, where appropriate responses are defined as in Experiment 3. Analysis of simple effects revealed that appropriate responding to NP cues was significantly greater than chance in every block [for Block 1, $F(1,20)=6.45, p<$ .05 ; for all other blocks, smallest $F(1,20)=20.53, p<$ $.001]$. As in Experiment 3, these data demonstrate that the participants learned about the partial predictiveness of NP cues and responded on the basis of the identity of these cues when they were presented (rather than guessing).

Figure 11A shows mean meal ratings across Stage 2. These meal ratings are calculated such that, for each participant, a choice of the pole of the scale labeled "Sure to be fish and chips" corresponds to a rating of 0 and a choice of the pole labeled "Sure to be burger and chips" corresponds to a rating of 40. An ANOVA with factors of predictiveness, valence, and block (omitting Block 1 data) revealed a significant main effect of valence $\left[F(1,20)=118.86, M S_{\mathrm{e}}=510.06\right]$. Discrimination between cues paired with profits and those paired with losses improved across Stage 2 training, and the block $\times$ valence interaction demonstrates that this trend was significant $\left[F(4,80)=39.09, M S_{\mathrm{e}}=36.54\right]$. Crucially, there was a significant interaction between predictiveness and 


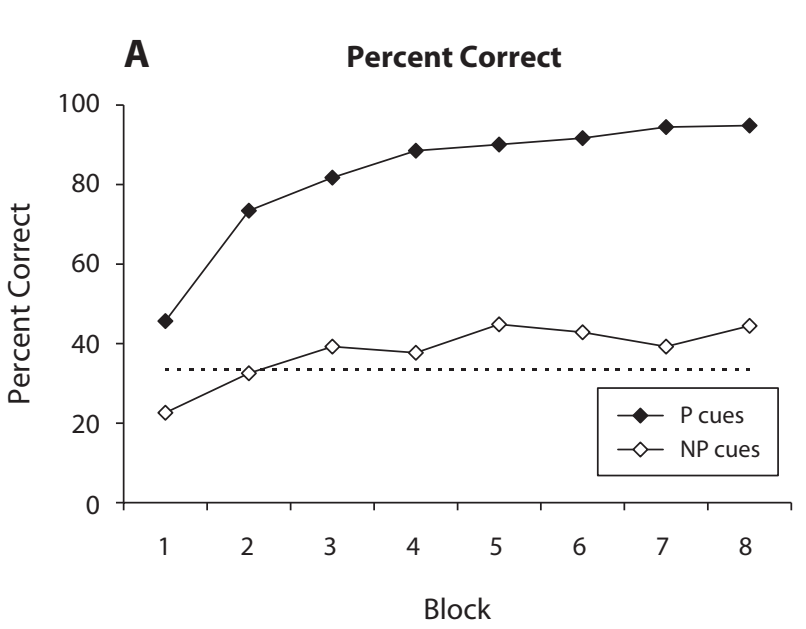

B

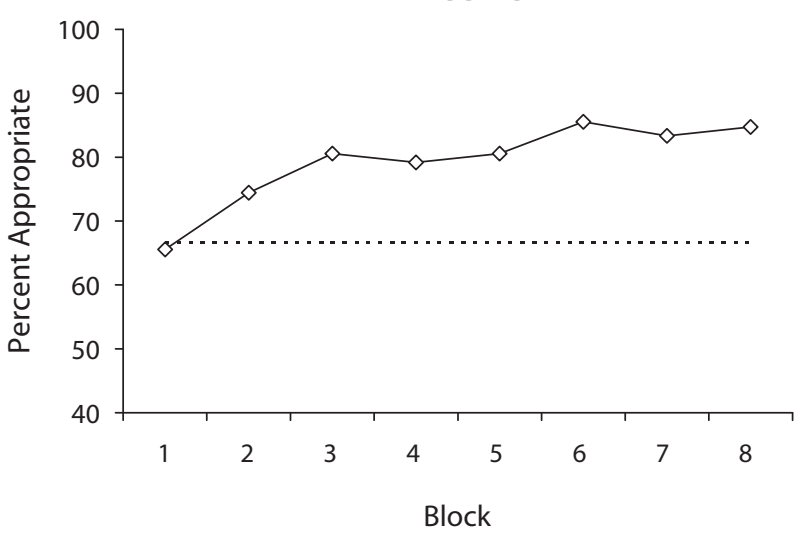

Figure 10. (A) Mean percentages of correct responses across Stage 1 in Experiment 4, averaged separately for predictive (P) cues and nonpredictive (NP) cues. The dotted line shows the theoretical level of chance responding $(33.3 \%)$. (B) Mean percentages of appropriate responses to NP cues across Stage 1 in Experiment 4, where a response was deemed appropriate if the participant chose an outcome that could feasibly be paired with that NP cue.

valence $\left[F(1,20)=4.59, M S_{\mathrm{e}}=62.38\right]$; given the pattern of means in Figure $11 \mathrm{~A}$, this interaction indicates that there was significantly better discrimination between $\mathrm{P}$ cues paired with the different meals than between NP cues.

Figure 11B shows discrimination scores for these cues. These were calculated for $\mathrm{P}$ cues by subtracting the mean meal rating received by $\mathrm{P}$ cues that were paired with fish and chips (A, B, and $\mathrm{C}$ ) from that received by $\mathrm{P}$ cues that were paired with burger and chips (D, E, and F). An equivalent score was calculated for NP cues. Figure 11B shows a clear discrimination advantage for $\mathrm{P}$ cues over NP cues, especially early in Stage 2 training. A paired $t$ test revealed that the mean discrimination score across Blocks 2-6 for $\mathrm{P}$ cues was significantly higher than that for NP cues $[t(20)=2.14]$. This again indicates better learning of the discrimination involving $\mathrm{P}$ cues than of that involving NP cues.
The Stage 2 rating data imply that we were successful in generating Stage 2 outcomes that were evaluatively equivalent. Notably, Figure 11A reveals that there was no hint of a general difference in ratings for $\mathrm{P}$ cues, as compared with NP cues, in Block $1(t<1)$. This contrast assesses the possibility of a bias that was consistent across all the participants. However, it is also possible that the bias was idiosyncratic to individual participants. That is, half of the participants might have viewed "fish and chips" more positively than "burger and chips," whereas the other half viewed "burger and chips" as more positive than "fish and chips." If the participants tended to rate P cues as more likely to be associated with the more positive outcome,
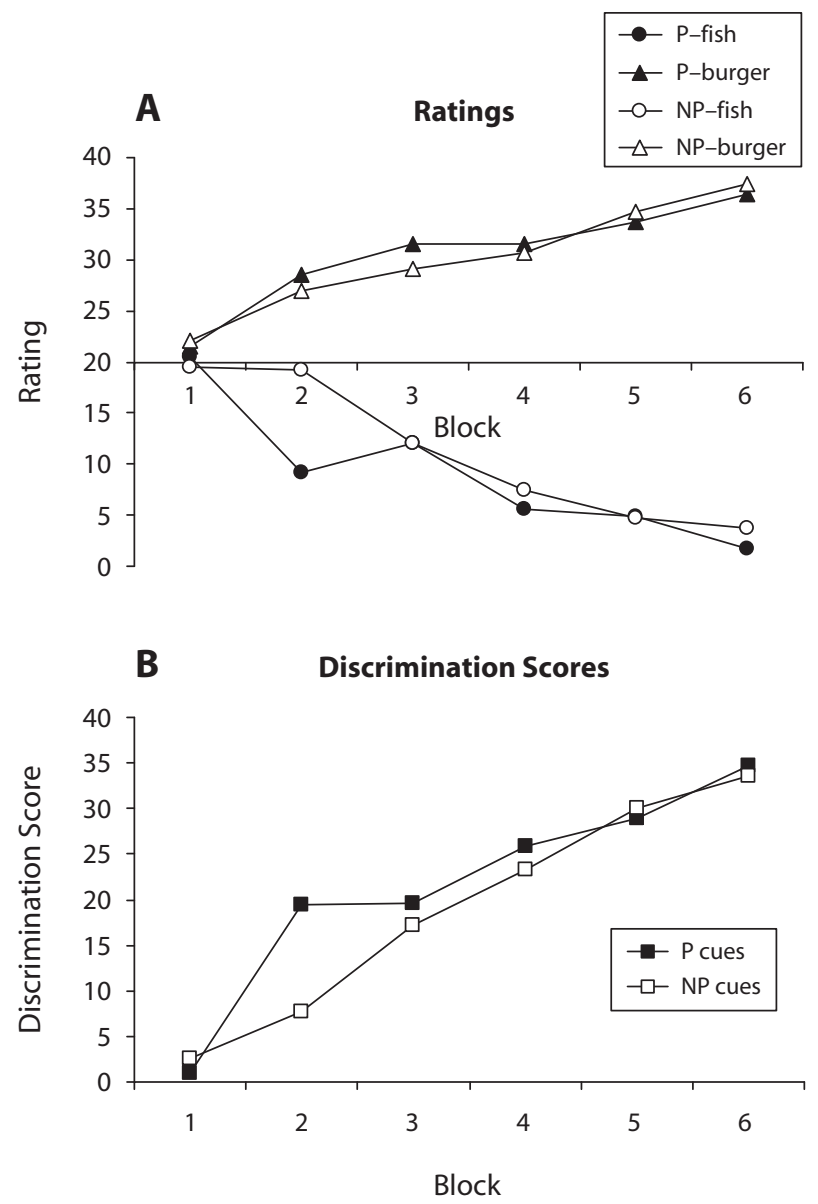

Figure 11. (A) Mean meal ratings across Stage 2 in Experiment 4. Ratings are calculated such that, for each participant, a choice of the pole of the scale labeled "Sure to be fish and chips" corresponds to a rating of 0 and a choice of the pole labeled "Sure to be burger and chips" corresponds to a rating of 40. Ratings are averaged separately for previously predictive $(P)$ cues paired with fish and chips during Stage 2 ( $P$-fish), previously $P$ cues paired with burger and chips (P-burger), previously nonpredictive (NP) cues paired with fish and chips (NP-fish), and previously NP cues paired with burger and chips (NP-burger). (B) Mean discrimination scores for Stage 2 in Experiment 4, averaged separately for $P$ cues and NP cues. These scores are calculated by subtracting the mean meal rating received by companies that were paired with fish and chips from that received by companies that were paired with burger and chips. 
averaging across all the participants would reveal no consistent pattern, and yet the individual bias held by each participant could feasibly have driven better discrimination of $P$ cues than of NP cues. If this were the case, we should expect those participants showing a larger bias in either direction at the outset of Stage 2 to show a greater difference in discrimination of P cues and NP cues. In other words, if this general bias was the source of the difference in discrimination learning, the participants who saw one meal as much more positive than the other (i.e., the participants who showed a large bias) should show a larger difference in discrimination learning than should the participants who viewed the two meals as evaluatively equivalent. For each participant, we calculated (1) the absolute value of the difference in mean ratings of $\mathrm{P}$ and NP cues during Block 1 of Stage 2 and (2) the difference in discrimination scores for $P$ and NP cues, averaged across Blocks 2-6 of Stage 2 (cf. Figure 11B). Contrary to the positive correlation anticipated if a general bias were the driving force behind the difference in discrimination of $\mathrm{P}$ and NP cues, this correlation was negative and nonsignificant $[r(21)=-.17, p=$ .47]. If, instead, we use the absolute value of the difference in mean rating of $\mathrm{P}$ and NP cues averaged across all the blocks of Stage 2 (rather than focusing only on Block 1), the correlation is near zero $[r(21)=.013, p=.96]$.

The results of Experiment 4 therefore imply that the better discrimination of $\mathrm{P}$ cues that were paired with different outcomes during Stage 2, as compared with NP cues that were paired with different outcomes, did not depend on an initial difference in the ratings of these classes of cues. Instead, this difference in discrimination seems to reflect a difference in the rate of learning about these classes of cues across Stage 2 training.

\section{GENERAL DISCUSSION}

Four experiments using human participants demonstrated better discrimination learning about cues previously experienced as predictive than about those previously experienced as nonpredictive, when this prior predictiveness was established via single-cue training. This finding indicating positive transfer of alpha is at odds with the suggestion (Le Pelley, 2004) that pretraining procedures emphasizing differences in absolute predictiveness, as compared with relative predictiveness, will tend to produce negative transfer of alpha. In the case of human contingency learning, it would seem that there is always a learning advantage for consistent predictors, regardless of whether cues are trained individually or as part of a compound.

Experiment 2 attempted to rule out an explanation of this finding in terms of differences in proactive interference by equating the number of sources of interference for P and NP cues. All the cues were paired with each of the different outcomes equally often during Stage 1. For $P$ cues, these pairings were blocked across training (i.e., all the pairings of a cue with one outcome were experienced before all the pairings with the other outcome), whereas for NP cues, the pairings were intermixed (such that a cue could be paired with each of the outcomes throughout Stage 1). This is clearly not a perfect control, given that the amount of interference affecting Stage 2 learning might be influenced by this trial order manipulation. For example, in the final block of Stage 1 training that immediately precedes Stage 2, each NP cue would be paired with two different outcomes, whereas each P cue would be paired with the same outcome twice. Consequently, any influence of recency or primacy could feasibly produce differences in the effective amount of interference experienced by these cues. In practical terms, however, it is not clear how one might control for interference more effectively in a singlecue training design. Moreover, it is not easy to see why prior learning about colors of business cards would interfere with learning about companies' profitability. This is particularly the case given that participants are unable to directly express their knowledge of card colors in Stage 2 (the only response options in Stage 2 relate to profitability), and hence, there is no way for responses learned in Stage 1 to interfere with acquisition of novel responses in Stage 2. Consequently, it is perhaps unsurprising that the advantage for $\mathrm{P}$ cues over NP cues should survive the manipulation in Experiment 2.

In Wilson et al.'s (1992) animal study, the individually pretrained light was, in both groups, the best available predictor of the tone (even if it was an inconsistent predictor in Group Experimental), such that a relative predictiveness mechanism would be bound to select the light for attention. This was not the case, however, in Experiments 1 and 2, where the particular Stage 1 trial structure meant that the experimental context was a marginally better predictor of the outcome on trials involving an NP cue than was the cue itself. Hence, the learning deficit for NP cues in these experiments could potentially still reflect the operation of a relative predictiveness mechanism. Experiment 3 ruled out this suggestion, using a three-outcome design in Stage 1 in which NP cues were partially predictive: They did not predict which outcome would occur on a given trial but did predict which outcome would not occur. Analysis of participants' choices on NP trials made it clear that they had indeed learned about the partial predictiveness of these cues and, by extension, had learned that, on these trials, the NP cues were the best available predictors of reinforcement: the participants clearly selected the NP cues as the information on which to base their predictions. An advantage for $\mathrm{P}$ cues over NP cues in Stage 2 learning was still observed, indicating that differences in cue selection on the basis of relative predictiveness were not the source of this effect.

Finally, Experiment 4 demonstrated that the Stage 2 learning advantage for $\mathrm{P}$ cues over NP cues did not depend on there existing an initial, general bias in participants' ratings regarding the two sets of cues. The implication is that this learned predictiveness effect reflects a difference in the rate at which cue-outcome associations are acquired for $\mathrm{P}$ cues and NP cues.

\section{Alpha Versus Outcome-Mediated Acquired Distinctiveness}

In the introduction, we noted that prior studies of alpha effects in human contingency learning have established predictiveness in a pretraining phase involving multiple 
simultaneously presented cues (e.g., Bonardi et al., 2005; Griffiths \& Le Pelley, 2009; Kruschke, 1996; Le Pelley \& McLaren, 2003; Livesey \& McLaren, 2007; Lochmann \& Wills, 2003; Maes et al., 2004). That said, there do exist prior studies of the transfer of learning following individual cue pretraining in humans (e.g., Hall et al., 2003; Norcross, 1958; Reese, 1972; for a review, see Hall, 1991). These studies have demonstrated that, if two different stimuli are consistently paired with two different outcomes in Phase 1 (i.e., $\mathrm{A} \rightarrow$ Outcome 1 and B $\rightarrow$ Outcome 2), subsequent learning of a discrimination between $\mathrm{A}$ and $\mathrm{B}$ with respect to new outcomes in Phase 2 (A $\rightarrow$ Outcome 3 and $\mathrm{B} \rightarrow$ Outcome 4 ) proceeds more rapidly than if $\mathrm{A}$ and $\mathrm{B}$ had been paired with the same outcome in Phase 1. In a sense, then, such studies demonstrate positive transfer; learning that $\mathrm{A}$ and $\mathrm{B}$ predict distinct outcomes in Phase 1 aids learning of the fact that $\mathrm{A}$ and $\mathrm{B}$ predict distinct outcomes in Phase 2. However, there are two important (and related) differences between these prior examples of positive transfer and the positive transfer demonstrated in the present experiments. First, the critical comparison is different in each case. In the present experiments, we compared the rate of discrimination learning for previously predictive cues with that for previously nonpredictive cues. In the prior studies listed above, however, both $\mathrm{A}$ and $\mathrm{B}$ were predictive during Phase 1 in both conditions of the experiment; the experimental manipulation regarded whether they predicted the same outcome or different outcomes during Phase 1. This leads on to the second difference - namely, that this design leaves these prior studies open to explanation in terms of outcome-mediated processes of acquired distinctiveness. The central idea is that presentation of Cues A and B in Phase 2 will evoke representations of their associates from Phase 1 (namely, Outcomes 1 and 2), and these associates can themselves enter into association with the Phase 2 outcomes. If A and B are paired with different associates in Phase 1, retrieval of these associates will aid Phase 2 discrimination learning, since the effective pattern of stimulation on each Phase 2 trial (either "A plus evoked Outcome 1" or "B plus evoked Outcome 2") is rendered more distinct than if A and B were paired with the same associate in Phase 1 (such that the effective pattern of stimulation on each Phase 2 trial is either "A plus evoked Outcome 1" or "B plus evoked Outcome 1"). For a more detailed discussion of this class of account, the reader is referred to Hall (1991, Chap. 5). There seems little doubt that such an outcome-mediated associative mechanism will contribute to the positive transfer that is demonstrated in these studies; the evidence in support of such a mechanism is clear (Hall et al., 2003). Consequently, the transfer demonstrated in these studies tells us little about the influence of prior experience on the alpha of the cues involved, which was the focus of the present experiments.

Crucially, as was noted in the introduction to Experiment 1 , the statistical independence of the outcomes in Stages 1 and 2 of the present experiments rules out any explanation of differences in learning about $\mathrm{P}$ and NP cues in terms of outcome-mediated acquired distinctiveness mechanisms. A similar argument applies to the other studies listed above as providing evidence for the positive transfer of alpha following multiple-cue pretraining (Bonardi et al., 2005; Griffiths \& Le Pelley, 2009; Kruschke, 1996; Le Pelley \& McLaren, 2003; Livesey \& McLaren, 2007; Lochmann \& Wills, 2003; Maes et al., 2004). Of the set of studies using humans that is not amenable to analysis in terms of outcome-mediated associative processes, the present experiments represent, to the best of our knowledge, the first to investigate the influence of individual-cue pretraining on alpha.

Interestingly, there is a parallel situation in the animal literature. That is, there exist studies demonstrating that prior training in which Cues A and B are paired with different outcomes will enhance subsequent discrimination between these cues, as compared with prior training in which A and B are paired with the same outcome (e.g., Honey \& Hall, 1989; Spear, Kraemer, Molina, \& Smoller, 1988; Spear \& Molina, 1987; for a review, see Hall, 1991). Once again, however, such studies are open to interpretation in terms of the operation of an outcome-mediated process of acquired distinctiveness, and there is good evidence to suggest that this is the correct interpretation of such effects (Honey \& Hall, 1989). There exists a further set of animal studies to which this account is not easily applied but that also involve pretraining with individual cues, each of which is the best available predictor of the outcome with which it is paired. Notably, and unlike in the experiments with humans reported here, these studies have typically shown evidence consistent with negative transfer of alpha - that is, faster learning about cues that were previously inconsistent predictors, as opposed to consistent predictors (e.g., Hall \& Pearce, 1979, 1982; Haselgrove et al., in press; Swan \& Pearce, 1988; Wilson et al., 1992). ${ }^{5}$

\section{Alpha in Animals and Humans}

Hence, we might tentatively argue that there exists a discrepancy between studies of alpha processes in humans and animals - that is, the set of studies in which outcomemediated acquired distinctiveness processes do not provide a viable account of the transfer effects that are observed. In animal studies, individual cue pretraining tends to produce negative transfer of alpha, whereas the present experiments indicate that, in humans, individual-cue pretraining produces positive transfer of alpha.

Clearly, in the absence of further evidence, this suggestion is speculative. Nevertheless, if we suppose for the sake of argument that it is correct, the question becomes one of why there is a discrepancy between the results of the present experiments and those of analogous animal studies. One possibility relates to the fact that the present experiments manipulated predictiveness on a withinsubjects basis, whereas analogous studies of alpha effects in animals involving individual-cue pretraining have used a between-subjects manipulation (e.g., Wilson et al., 1992). A within-subjects design allows the possibility of a comparison of the relative predictiveness of cues across trials. That is, when a particular cue is presented, participants might compare the predictiveness of that cue with the predictiveness of other cues that they have experienced 
on previous trials. This comparison will reveal $\mathrm{P}$ cues to be more predictive than NP cues. Such a relative comparison across trials could then underpin the advantage for $P$ cues in Stage 2. It is worth noting that this suggestion of comparison across trials is very different from the process envisaged by existing attentional models (e.g., Kruschke, 1996, 2001; Mackintosh, 1975), which rely on a comparison of the relative ability of simultaneously presented cues to predict the particular outcome that has occurred on a trial. This raises the question of how a comparison across trials could be instantiated. It is not easy to see how it could rely on a comparison of the ability of different cues to predict the same, specific outcome, since, on this basis, certain comparisons would reveal all cues to be relatively good predictors (e.g., Cue X in Experiment 1 is paired with pink and orange and is, therefore, a better predictor of orange than is Cue A, which is always paired only with pink). Rather, the comparison would have to be in terms of some more abstract notion of predictiveness relative to outcomes in general, rather than to one outcome in particular. A further problem for this account comes in working out exactly which cues ought to be compared. Clearly, a comparison of two different P cues (or two different NP cues) would reveal neither to be more predictive than the other. An alternative would be to specify that comparison is between a $\mathrm{P}$ cue and an NP cue, but for participants to pick out such a comparison presupposes that they understand the difference in predictiveness of the two cue classes, and hence the argument becomes circular. A more practicable approach might involve a comparison of the predictiveness of the cue presented on a particular trial with the average predictiveness of all other cues encountered thus far.

Regardless of the best way to instantiate an across-trials comparison of predictiveness, the approach described above supposes that the key difference between animal and human studies is in experimental design, rather than in there being a fundamental difference in the influence of learned predictiveness in the two cases. Of course, this idea could be tested by conducting a within-subjects study of learned predictiveness effects following single-cue training in animals. In fact, Wilson et al.'s (1992) Experiment 3 does manipulate predictiveness within subjects. However, in contrast to Experiments 1 and 2 from that article, this experiment merely examined the influence of this manipulation on the magnitude of the orienting response elicited by the cues involved and did not test the rate of novel learning about these cues when they were paired directly with the food unconditioned stimulus. Consequently, this study reveals nothing about how this within-subjects manipulation of predictiveness influenced the alpha of the cues.

An alternative would be to manipulate predictiveness on a between-subjects basis in human participants. We have resisted this approach, given the difficulties of interpretation that would result from the possibility of between-group differences that are unrelated to alpha. For example, it is easy to imagine that experience of a "solvable" training phase involving only predictive cues, as compared with an "unsolvable" phase involving only nonpredictive cues, would give rise to motivational differences between groups that would transfer to influence the rate of learning during Stage 2. Of course, this possibility could feasibly also apply to the previously mentioned between-subjects studies with animals (cf. Winefield, 1978) and, hence, provides another way of reconciling the present results with those in animal conditioning research, by ascribing the results of these animal studies to between-group differences in, say, motivation, rather than to a difference in alpha. This emphasizes the importance of establishing whether similar effects can be observed in within-subjects studies in animals.

If the difference between the results of the animal and human studies is not simply a consequence of experimental methodology, then (still assuming that this difference is real and meaningful) this suggests the possibility of a difference in the operation of alpha mechanisms in humans and animals. In fact, this suggestion is not without precedent. It has been noted many times that animals and humans differ in their propensity to show latent inhibition, the detrimental effect of nonreinforced exposure to a cue on the rate of subsequent conditioning to that cue (e.g., Graham \& McLaren, 1998; Lubow, 1973; Lubow \& Gewirtz, 1995). Latent inhibition is often viewed as reflecting a reduction in the alpha of the preexposed stimulus during nonreinforced preexposure (which is typically conducted with that stimulus presented in isolation) and follows naturally from the Pearce-Hall model (although alternative accounts have also been advanced; see Hall, 1991). Whereas findings of latent inhibition in animal studies are ubiquitous (see Lubow, 1989), analogous studies with humans have typically failed to reveal a similar effect, and those studies that have claimed to demonstrate "simple" latent inhibition in humans are typically open to alternative explanation (Graham \& McLaren, 1998). As in the present experiments, then, this interpretation sees latent inhibition as an alpha effect arising from singlecue pretraining that differs between humans and nonhuman animals. Moreover, those studies that have reported a latent-inhibition-like effect in humans have almost invariably used a masking task during preexposure, which functions to divert attention away from the preexposed stimulus. Consequently, use of a masking task allows relative predictiveness processes to operate, by creating a situation more akin to pretraining with multiple simultaneously presented cues that differ in their predictiveness (Le Pelley \& Schmidt-Hansen, in press). That is, the preexposed stimulus is less predictive of the response to be made on the masking task (being entirely irrelevant to that task) than are the stimuli of the masking task themselves, and hence, selective attention (operating on the basis of relative predictiveness, much as suggested by Mackintosh, 1975) would tend to move away from the preexposed stimulus (similar analyses are suggested by Graham \& McLaren, 1998, and Kruschke, 2001). Given that, as was noted earlier, it is well established that humans tend to show a retardation in learning about less predictive cues following multiple-cue pretraining, it is therefore unsurprising that slower learning about the preexposed cue is observed under these conditions. 


\section{Conclusion}

The present experiments indicate that, in human associative learning, prior experience of the predictiveness of cues leads to more rapid learning about cues experienced as more predictive than about those experienced as less predictive, even when that predictiveness is established via training with individual cues, each of which is the best available predictor of the outcome with which it is paired. The implication is that alpha in these experiments was influenced by the absolute predictiveness of cues, with a higher alpha for cues with a higher absolute predictiveness. This raises the possibility that previously reported learned predictiveness effects observed in humans following pretraining with multiple simultaneously presented cues might reflect a difference in the absolute predictiveness of the cues involved, rather than a comparison of relative predictiveness, as is often assumed by attentional accounts of these effects (e.g., Kruschke, 2001, 2006; Mackintosh, 1975). Of course, it is quite possible that differences in relative predictiveness also contribute to the advantage observed for more predictive cues over less predictive cues in these previous studies. Thus, whether learned predictiveness effects in human associative learning are subserved by one alpha system (based on absolute predictiveness) or two separate alpha systems (based on absolute and relative predictiveness) remains to be established.

\section{AUTHOR NOTE}

This work was supported by Grant RES000230983 from the Economic and Social Research Council to M.E.L. The authors thank Tom Beesley for his comments on a draft of the manuscript. Correspondence concerning this article should be addressed to M. E. Le Pelley, School of Psychology, Cardiff University, Cardiff CF10 3AT, Wales (e-mail: lepelleyme@cardiff.ac.uk).

\section{REFERENCES}

Bennett, C. H., Wills, S. J., OAKeshott, S. M., \& Mackintosh, N. J. (2000). Is the context specificity of latent inhibition a sufficient explanation of learned irrelevance? Quarterly Journal of Experimental Psychology, 53B, 239-253.

Bonardi, C., Graham, S., Hall, G., \& Mitchell, C. (2005). Acquired distinctiveness and equivalence in human discrimination learning: Evidence for an attentional process. Psychonomic Bulletin \& Review, 12, 88-92.

De Houwer, J., Beckers, T., \& Vandorpe, S. (2005). Evidence for the role of higher order reasoning processes in cue competition and other learning phenomena. Learning \& Behavior, 33, 239-249.

Dickinson, A. (2001). Causal learning: An associative analysis. Quarterly Journal of Experimental Psychology, 54B, 3-25.

Dopson, J., Esber, G. R., \& Pearce, J. M. (in press). Differences between the associability of relevant and irrelevant stimuli are not due to acquired equivalence and distinctiveness. Journal of Experimental Psychology: Animal Behavior Processes.

George, D. N., \& Pearce, J. M. (1999). Acquired distinctiveness is controlled by stimulus relevance not correlation with reward. Journal of Experimental Psychology: Animal Behavior Processes, 25, 363-373.

GrahaM, S., \& MCLaren, I. P. L. (1998). Retardation in human discrimination learning as a consequence of pre-exposure: Latent inhibition or negative priming? Quarterly Journal of Experimental Psychology, 51B, 155-172

Griffiths, O., \& Le Pelley, M. E. (2009). Attentional changes in blocking are not a consequence of lateral inhibition. Learning \& Behavior, 37, 27-41.

Hall, G. (1991). Perceptual and associative learning. Oxford: Oxford University Press.

Hall, G., Mitchell, C. J., Graham, S., \& Lavis, Y. (2003). Acquired equivalence and distinctiveness in human discrimination learning: Evidence for associative mediation. Journal of Experimental Psychology: General, 132, 266-276.

Hall, G., \& Pearce, J. M. (1979). Latent inhibition of a CS during CS-US pairings. Journal of Experimental Psychology: Animal Behavior Processes, 3, 31-42.

Hall, G., \& Pearce, J. M. (1982). Changes in stimulus associability during conditioning: Implications for theories of acquisition. In M. L. Commons, R. J. Herrnstein, \& A. R. Wagner (Eds.), Quantitative analyses of behavior (Vol. 3, pp. 221-239). Cambridge, MA Ballinger.

Haselgrove, M., Esber, G. R., Pearce, J. M., \& Jones, P. M. (in press). Two kinds of attention in Pavlovian conditioning: Evidence for a hybrid model of learning. Journal of Experimental Psychology: Animal Behavior Processes.

Honey, R. C., \& Hall, G. (1989). The acquired equivalence and distinctiveness of cues. Journal of Experimental Psychology: Animal Behavior Processes, 15, 338-346.

KRUSCHKE, J. K. (1996). Dimensional relevance shifts in category learning. Connection Science, 8, 225-247.

KrUSCHKE, J. K. (2001). Towards a unified model of attention in associative learning. Journal of Mathematical Psychology, 45, 812-863.

KruschKe, J. K. (2006). Locally Bayesian learning with applications to retrospective revaluation and highlighting. Psychological Review, 113, 677-699.

Le Pelley, M. E. (2004). The role of associative history in models of associative learning: A selective review and a hybrid model. Quarterly Journal of Experimental Psychology, 57B, 193-243.

Le Pelley, M. E. (in press-a). Attention and human associative learning. In C. J. Mitchell \& M. E. Le Pelley (Eds.), Attention and associative learning: From brain to behaviour. Oxford: Oxford University Press.

Le Pelley, M. E. (in press-b). The hybrid modeling approach to conditioning. In N. A. Schmajuk (Ed.), Computational models of conditioning. Cambridge: Cambridge University Press.

Le Pelley, M. E., \& McLaren, I. P. L. (2003). Learned associability and associative change in human causal learning. Quarterly Journal of Experimental Psychology, 56B, 68-79.

Le Pelley, M. E., \& Schmidt-Hansen, M. (in press). Latent inhibition and learned irrelevance in human contingency learning. In R. E. Lubow \& I. Weiner (Eds.), Latent inhibition: Cognition, neuroscience, and applications to schizophrenia. Cambridge: Cambridge University Press.

Le Pelley, M. E., Schmidt-Hansen, M., Harris, N. J., Lunter, C. M., \& Morris, C. S. (2010). Disentangling the attentional deficit in schizophrenia: Pointers from schizotypy. Psychiatry Research, 176, 143-149.

Livesey, E. J., \& McLaren, I. P. L. (2007). Elemental associability changes in human discrimination learning. Journal of Experimental Psychology: Animal Behavior Processes, 33, 148-159.

Lochmann, T., \& Wills, A. J. (2003). Predictive history in an allergy prediction task. In F. Schmalhofer, R. M. Young, \& G. Katz (Eds.), Proceedings of EuroCogSci 03 (pp. 217-222). Mahwah, NJ: Erlbaum.

López, F. J., Shanks, D. R., Almaraz, J., \& Fernández, P. (1998). Effects of trial order on contingency judgments: A comparison of associative and probabilistic contrast accounts. Journal of Experimental Psychology: Learning, Memory, \& Cognition, 24, 672-694.

Lubow, R. E. (1973). Latent inhibition. Psychological Bulletin, 79, 398407.

Lubow, R. E. (1989). Latent inhibition and conditioned attention theory. Cambridge: Cambridge University Press.

Lubow, R. E., \& GewirTz, J. C. (1995). Latent inhibition in humans: Data, theory, and implications for schizophrenia. Psychological Bulletin, 117, 87-103.

Lubow, R. E., \& Moore, A. U. (1959). Latent inhibition: The effect of nonreinforced preexposure to the conditional stimulus. Journal of Comparative \& Physiological Psychology, 52, 415-419.

Mackintosh, N. J. (1969). Further analysis of the overtraining reversal effect. Journal of Comparative \& Physiological Psychology, 67(Suppl.), 1-18.

MaCKINTOSH, N. J. (1973). Stimulus selection: Learning to ignore stimuli that predict no change in reinforcement. In R. A. Hinde \& J. S. Hinde (Eds.), Constraints on learning (pp. 75-96). London: Academic Press. Mackintosh, N. J. (1975). A theory of attention: Variations in the as- 
sociability of stimuli with reinforcement. Psychological Review, 82, 276-298.

Mackintosh, N. J., \& LitTLe, L. (1969). Intradimensional and extradimensional shift learning by pigeons. Psychonomic Science, 14, 5-6.

Mackintosh, N. J., \& TuRner, C. (1971). Blocking as a function of novelty of CS and predictability of UCS. Quarterly Journal of Experimental Psychology, 23, 359-366.

Maes, J. H. R., Damen, M. D. C., \& Eling, P. A. T. M. (2004). More learned irrelevance than perseveration errors in rule shifting in healthy subjects. Brain \& Cognition, 54, 201-211.

McGeoch, J. A., \& McDonald, W. T. (1931). Meaningful relation and retroactive inhibition. American Journal of Psychology, 43, 579-588.

NoRCROSS, K. J. (1958). Effects on discrimination performance of similarity of previously acquired stimulus names. Journal of Experimental Psychology, 56, 305-309.

Pearce, J. M., \& Hall, G. (1980). A model for Pavlovian conditioning: Variations in the effectiveness of conditioned but not of unconditioned stimuli. Psychological Review, 87, 532-552.

Pearce, J. M., \& Mackintosh, N. J. (in press). Two theories of attention: A review and a possible integration. In C. J. Mitchell \& M. E. Le Pelley (Eds.), Attention and associative learning: From brain to behaviour. Oxford: Oxford University Press.

REESE, H. W. (1972). Acquired distinctiveness and equivalence of cues in young children. Journal of Experimental Child Psychology, 13, 171-182.

ShapIRo, S. S., \& WiLK, M. B. (1965). An analysis of variance test for normality (complete samples). Biometrika, 52, 591-611.

Spear, N. E., Kraemer, R. J., Molina, J. C., \& Smoller, D. E. (1988). Developmental change in learning and memory: Infantile disposition for unitization. In J. Delacour \& J. C. S. Levy (Eds.), Systems with learning and memory abilities (pp. 27-52). Amsterdam: Elsevier.

SPEAR, N. E., \& Molina, J. C. (1987). The role of sensory modality in the ontogeny of stimulus selection. In N. Krasnegor, E. M. Blass, M. A. Hofer, \& W. P. Smotherman (Eds.), Perinatal development: A psychobiological perspective (pp. 83-110). Orlando, FL: Academic Press.

Sutherland, N. S., \& Mackintosh, N. J. (1971). Mechanisms of animal discrimination learning. New York: Academic Press.

Swan, J. A., \& Pearce, J. M. (1988). The orienting response as an index of stimulus associability in rats. Journal of Experimental Psychology: Animal Behavior Processes, 14, 292-301.

Wilson, P. N., Boumphrey, P., \& Pearce, J. M. (1992). Restoration of the orienting response to a light by a change in its predictive accuracy. Quarterly Journal of Experimental Psychology, 44B, 17-36.

WinEFIELD, A. H. (1978). The effect of prior random reinforcement on brightness discrimination learning in rats. Quarterly Journal of Experimental Psychology, 30, 113-119.

\section{NOTES}

1. Hence, there were four P cues but only two NP cues during Stage 1 training in Experiment 1. Since the participants could achieve, at best,
$50 \%$ correct responses for these cues on average (as compared with $100 \%$ for P cues), the higher the proportion of NP cues, the lower the mean percent correct would be during Stage 1. Given the potential of a low overall percentage of correct responses to demotivate participants, our aim was therefore to minimize the number of NP cues. However, any concerns that are raised by the imbalance in the number of $\mathrm{P}$ and NP cues in Experiments 1 and 2 should be allayed by the fact that equal numbers of each were used in Experiments 3 and 4.

2. A similar argument applies even if we merely specify that, across Stage $1, X$ should be paired with pink six times and orange six times and then shuffle these presentations randomly (thereby removing the restriction to run lengths of the same outcome of no more than two). Suppose that, on its first presentation, $\mathrm{X}$ is paired with pink. If participants base their predictions on their previous experience, then on the next presentation of $\mathrm{X}$, they might be inclined to again predict pink. However, the probability of the correct answer's being pink on this second presentation of $X$ would be $5 / 11=.45$, and so the participants would be more likely to be incorrect than correct. A similar argument applies to subsequent presentations of $\mathrm{X}$ and will tend to generate below-chance performance across the whole of Stage 1

3. In all the other experiments reported in this article, the corresponding set of differences in mean discrimination scores did not differ significantly from a normal distribution ( $p$ s $>.1)$, and hence, parametric statistics were used for the corresponding analyses.

4. There is a severe restriction in the values that the independent variable can take for each participant. For the "NP+ vs. NP-" type (which is based on the response to a single comparison), the value can be either 0 or 1 . For the other types (which are all based on an average of responses to two equivalent comparisons), this value can be $0, .5$, or 1 . Given this restriction, a sign test is the most appropriate statistical procedure to use with these data.

5. An apparent exception to this pattern is the demonstration in animals of "simple" learned irrelevance, in which previous experience of an individual cue as being uncorrelated with an outcome leads to a retardation in subsequent learning about that cue (e.g., Mackintosh, 1973). Crucially, however, the cue in such experiments is a poorer predictor of the outcome than is the experimental context (much as in the present Experiments 1 and 2): The outcome can occur in the absence of the cue, but never in the absence of the context. Consequently, the context can be treated as an additional, simultaneously presented cue. Indeed, the "standard" analysis of this effect in terms of attentional theories, such as the Mackintosh model, relies on a comparison of relative predictiveness between cue and context. In contrast, in animal studies demonstrating negative transfer of associability (e.g., Wilson et al., 1992), the individually pretrained cue is a better predictor of the outcome than is the context, so there is no scope for a comparison of relative predictiveness to lead to a detriment in learning about that cue.

(Manuscript received May 23, 2009;

revision accepted for publication November 2, 2009.) 